\title{
Traducción del teatro de Fogazzaro: Il ritratto mascherato. El retrato de la máscara
}

\author{
Fernando Molina Castillo \\ Universidad de Sevilla \\ fernanmolina@us.es
}

\section{Roberto Randaccio}

Cagliari

randax58@tiscali.it

\begin{abstract}
La produzione teatrale di Antonio Fogazzaro è stata spesso trascurata ed emarginata dagli studi sull'autore. In quest'articolo si presentano, attraverso un percorso di testimonianze tratte dal suo epistolario, le vicende antecedenti e le motivazioni che portarono lo scrittore vicentino ad avvicinarsi al mondo della letteratura teatrale, con tre brevi opere, la seconda delle quali, Il ritratto mascherato, si presenta qui in edizione critica e, per la prima volta, in traduzione spagnola. Nel suo complesso l'esperienza teatrale fu valutata, a suo tempo, come un chiaro fallimento per lo scrittore, ma per lo studioso dell'opera fogazzariana, al contrario, risulta una fonte interessantissima di studio e approfondimento, soprattutto per i forti legami con i temi e le idee presenti nella sua opera narrativa.
\end{abstract}

Parole chiavi: Fogazzaro; Il ritratto mascherato; Teatro; Ottocento; traduzione spagnola.

\begin{abstract}
The dramatic works of Antonio Fogazzaro have frequently been neglected and marginalized in the studies on the author. This article, reviewing the testimonies found in the author's collection of letters, presents all the previous circumstances and motivations that led the writer from Vicenza to approach the world of theatrical literature, by means of his three short pieces, the second of which, Il ritratto mascherato, is presented here in the critical edition, and for the first time, in Spanish translation. Fogazzaro's theatrical achievements, taken as a whole, were at that time perceived as his complete failure as a playwright. In contrast, to Fogazzaro scholars they represent an interesting and rich research source, primarily for their strong links with the themes and ideas present in his narrative works.
\end{abstract}

Keywords: Fogazzaro; The portrait with the mask; Theatre; XIXth century; Spanish translation. 


\section{Una debole compiacenza. L'avventura teatrale di Antonio Fogazzaro}

In una lettera indirizzata all'amico Tommaso Gallarati Scotti, Antonio Fogazzaro definisce così la sua seconda opera teatrale appena conclusa: «E ora in fretta e furia Le dirò che in questi ultimi giorni ho scritto un altro drammino Il ritratto mascherato». ${ }^{1}$ Qualche tempo prima, riferendosi al suo primo lavoro teatrale, Il garofolo rosso, scriveva a Giuseppe Giacosa: «Però non m'illudo; uno spunto in dialetto è una cosa e una commedia italiana è un'altra»; $;^{2}$ per poi ribadire, sempre allo stesso: «Sono molto esitante a comparire sulle scene italiane con una simile pillola drammatica e dubito poi anche dell'effetto». ${ }^{3}$ "Drammino», "pillola drammatica», «spunto» dialettale, sono definizioni che attestano, da parte dello scrittore, una scarsa convinzione nelle proprie capacità d'autore teatrale. Di fatto, Fogazzaro non si sentiva pronto per il teatro, o, perlomeno, era cosciente della propria inesperienza: «Diventare autore drammatico a sessant'anni? Sarebbe un bel caso. Per quelle due inezie non mi considero tale», ${ }^{4}$ come ricordava ancora una volta all'amico Giuseppe Giacosa, che, al contrario, come vedremo, sosteneva con entusiasmo i suoi lavori e lo stimolava a «scrivere per il teatro». Una presa di coscienza delle proprie capacità, che confermava un concetto analogo espresso una ventina di anni prima: «L'altro dì ebbi una bellissima lettera dalla signora Duse. Mi scrive esprimendo il desiderio che Daniele Cortis venga ridotto per il teatro. Io non potrei, non vorrei farlo mai e non credo che un uomo d'ingegno possa accettare un compito simile. Se non lo fa l'autore, temo non lo voglia fare che un mestierante. Posso ingannarmi. Intanto risposi così alla signora Duse, la quale credo, del resto, che riuscirebbe una Elena vera». ${ }^{5}$ Primo segnale di una idiosincrasia alla scrittura teatrale che frena la fantasia dello scrittore vicentino, nonostante la "fascinazione» per il teatro, e soprattutto l'amicizia con Giacosa, i cui successi teatrali erano da Fogazzaro applauditi e condivisi. Qualche anno dopo ritornerà ancora sull'argomento, ben più deciso e radicato in opinioni di ferma opposizione. Ancora una volta è una lettera a Giacosa che testimonia il suo pensiero: «Non ho mai creduto possibile ridurre Cortis per il teatro. Credo averlo scritto anni sono alla signora Duse che mi proponeva di farlo. Lo avrei scritto al Ferri se mi avesse interpellato prima di scrivere il suo lavoro. No no

1. Lettere SCelte, n. 591 (Bergamo, 30 dicembre 1901), pp. 462-463.

2. Fogazzaro-Giacosa, n. 262 (Vicenza, 7 dicembre 1901), p. 323.

3. Ivi, n. 263 (Vicenza, 12 dicembre 1901), p. 324.

4. Ivi, n. 271 (Vicenza, 19 gennaio 1902), p. 331.

5. Lettere scelte, n. 77 (Vicenza, 11 luglio 1886), p. 111; vedi anche Fogazzaro-Giacosa, n. 56, p. 74. Oreste Palmiero, curatore del carteggio Fogazzaro-Giacosa, pubblica, in nota, la lettera della Duse (Varazze, 7 luglio 1886): «Certamente lo amate molto il vostro Daniele Cortis ed è tal libro che non va profanato. Io non so se è tutta arte, solamente, là dentro, so che tutto là dentro è buono, bello, forte e soavissimo. [...] ne soffrireste se a qualcuno venisse l'idea... di slegare le pagine, del dolce libro, serbate le passioni - riannodar tutto con mano forte - e dare ai personaggi un'altra forma d'arte - cioè: quella... del teatro?» (Fogazzaro-Giacosa, p. 74, nota 66). Si veda anche Piero Nardi, «Spunti fogazzariani», in Pègaso. Rassegna di lettere e arti, novembre 1932, pp. 617-621. 
no, io non so fare una commedia, ma sento come va fatta e sento che Daniele Cortis non può andare. Ho significato al signor Ferri il mio veto non senza un vivo rincrescimento perché quel pover'uomo ha buttato tempo, fatica e amore; del quale amore la mia gratitudine sincera gli è troppo scarso compenso». ${ }^{6}$ L'affermazione era categorica («no no no, io no so fare una commedia»), ma pur lasciava aperta una possibilità ("ma sento come va fatta»). In realtà, per quanto Fogazzaro affermi di non aver le doti per l'arte teatrale, prima ancora delle sue tre prove drammaturgiche d'inizio secolo, aveva composto un piccolo lavoro che, per quanto sia accorpato nel novero dei «racconti brevi», è strutturato come un vero e proprio piccolo atto unico. Si tratta de La lira del poeta, pubblicato la prima volta sulla rivista diretta da Enrico Panzacchi, Lettere e Arti, nel numero 13 del 12 aprile $1890 .^{7}$ La struttura narrativa è quella di una pièce teatrale, con tanto di didascalie sceniche e recitative; un bozzetto dove il dialetto veneto si mischia all'italiano, un testo rapido, spiritoso e pungente. Qui sta forse il segreto e l'ambizione, altrettanto segreta, per il teatro dello scrittore vicentino: la sua abilità nel costruire dialoghi vivaci e penetranti, facilmente mutabili in battute teatrali. ${ }^{8}$ Ancora, nella raccolta Fedele ed altri racconti (Milano, Galli, 1887), ritroviamo altri piccoli innesti teatrali, ossia quegli «intermezzi musicali» che si alternano tra un racconto e l'altro. Ci riferiamo in particolare al Terzo intermezzo ispirato al Minuetto in la di Boccherini; anche in questo caso abbiamo un impianto scenico ben definito dalle didascalie e una divisione delle dramatis personae secondo schemi prettamente teatrali (o melodrammatici).

L'avventura teatrale di Antonio Fogazzaro si compie quasi per caso, forzata dalle insistenze e dall'entusiasmo (forse un po' esagerato) i Giuseppe Giacosa, che spinge l'amico non solo a scrivere per il teatro, ma lo induce alla rappresen-

6. Lettere scelte, n. 273 (Vicenza, 8 ottobre 1892), p. 260, e Fogazzaro-Giacosa, n.153, pp. 191-192. Palmiero chiarisce che il «signor Ferri» citato nella lettera, ossia l'autore della riduzione teatrale del Daniele Cortis, sia un certo B. Ferri e non il «letterato romano» Giustino Ferri (1857-1913), come sostenuto dal Gallarati Scotti; cfr. Fogazzaro-Giacosa, p. 188, nota 175 e LetTERE SCElte, p. 260, nota 1. Palmiero, inoltre, riporta interamente la lettera che il signor «B. Ferri» indirizza allo scrittore vicentino per proporgli la propria riduzione del romanzo, trasformato in un dramma in cinque atti; si veda FogazzaroGiacosa, p. 192, nota 178. Giuseppe Giacosa, in una precedente lettera (5 ottobre 1892) precisava all'amico di aver letto la riduzione teatrale del Ferri, e, pur rispettando il lavoro e gli sforzi di quest'ultimo, dà un esplicito parere negativo: «Dunque ho trovato qui ieri il dramma Daniele Cortis. [...], eccoti il mio brutale parere: - No - [...]. Si possono ridurre a dramma i romanzi a grande ed anche intricato viluppo di fatti, non i romanzi interni e sottilmente scrutatori delle anime come è il tuo" (Fogazzaro-Giacosa, n. 152, p. 188). Si veda anche Enrico Ghidetti, Malattia, coscienza e destino. Per una mitografia del Decadentismo, Firenze: La Nuova Italia, 1993, p. 59.

7. Il racconto confluì, poi, nella raccolta Racconti brevi (Roma: Voghera editore, 1894), ed infine in Idilli spezzati. Racconti brevi (Milano: Baldini e Castoldi, 1902).

8. «Nei romanzi aveva sempre fatto un notevole uso dei dialoghi, e sapeva di avere ormai il possesso tecnico di uno strumento che gli avrebbe permesso di esprimere in forma dialogata qualsiasi concetto volesse» (Donatella e Leone PICCIOni, Fogazzaro, Torino: UTET, 1970, p. 357). 
tazione delle sue opere sul palcoscenico. Nell'autunno del 1900 Giacosa era stato chiamato da suo genero Albertini (allora direttore del Corriere della Sera) a dirigere $L a$ Lettura, rivista mensile del giornale milanese. Fin dal ricevimento dell'incarico, l'amico prega Fogazzaro di collaborare alla futura uscita del primo numero con un "pezzo» (un racconto o quant'altro). ${ }^{9}$ Fogazzaro, in quei mesi, è troppo impegnato nella messa a punto del testo a stampa di Piccolo mondo moderno, e non può sostenere le richieste dell'amico; passerà circa un anno prima che consegni uno scritto originale per la rivista. Si tratta del Garofolo rosso. ${ }^{10}$ Giacosa si dichiara subito entusiasta del testo: "Carissimo, ho letto ed ammirato. Quella piccola tragedia comica, mi ha colpito profondamente. Non credo convenga dividerla in scene per due ragioni: $1^{\mathrm{a}}$ perché, non destinata al teatro è inutile il richiamo continuo alla forma teatrale. $2^{\text {a }} \mathrm{E}$ specialmente per non rompere nemmeno con segni esteriori la continuità e l'unità di quella che è veramente scena unica. Del resto se tu stesso non lo hai fatto è segno che la pensi allo stesso modo». ${ }^{11}$ Il lavoro editoriale di correzione di bozze sarà rapidissimo e nel numero 12 della "Lettura" del dicembre 1901 vedrà le stampe il testo El garofolo rosso, sotto la dicitura esplicativa: «Spunti drammatici». A questo punto dobbiamo aggiungere due nuove definizioni alle precedenti:

9. «Ma intanto io sono senza novella per il primo numero [...]. Dunque? Dunque vinco la resistenza, mi rassegno alla indiscrezione e vengo a te colle mani giunte implorando soccorso. [...] Tu così gran signore dell'arte hai certo nei tuoi cassetti qualche novella abbozzata $\mathrm{e}$ incominciata" (FogazZAro-Giacosa, n. 256, Milano, 24 novembre 1900, p. 318).

10. La vicenda del Garofolo avviene tutta nella stanza dell'Ospizio per nobili infermi, dove l'anziana contessa Marieta, ormai cieca, vive reclusa, in uno stato permanente di loquace malumore; in particolare, ha una profonda repulsione verso i garofani che un'altra inferma coltiva in una stanza vicina. Quest'avversione è dovuta al brutto ricordo, che i fiori le suscitano, del suo ex marito Checo Busòlo, il quale, un mese prima di sposarla, aveva scommesso con un amico che avrebbe sedotto tre donne, tra cui lei, per mezzo di un solo garofano rosso. La donna chiama il custode per lamentarsi, ancora una volta, dell'odore dei garofani che invade la sua camera, e costui ne approfitta per informarla che anche il suo ex marito è stato ammesso a risiedere nell'Ospizio. Questa notizia scatena l'ira e il terrore nella contessa, la quale, però, non ha avvertito che, dietro al custode, è entrato anche Busòlo, che ascolta in silenzio le lamentele della donna, frugando nella stanza in cerca di qualcosa (ruberà una chiave da un cappotto della donna). La contessa pretende di parlare con il direttore dell'ospizio, che si trova a passare proprio da quelle parti. Il custode esce, ma Busòlo, in evidente combutta, resta in disparte, mentre il direttore ascolta le proteste della donna. Ma il direttore, a cui preme non perdere un appuntamento di gioco, esce dalla stanza alla chetichella lasciando Busòlo da solo, facendo intendere a quest'ultimo di farsi passare per lui. La contessa sfoga al finto direttore il suo rancore e il suoi timori nei confronti dell'ex marito. Accortasi finalmente dell'inganno, muore per la forte emozione. Nella confusione successiva, Busòlo, fingendosi addolorato, cerca di portar via ancora qualche cosa dalla stanza. Gli altri ospiti, cinicamente, valutano i vantaggi della nuova stanza ormai libera, mentre il custode si sente sollevato dal non dover più sopportare le lamentele della contessa. L'ultima perfidia nei confronti della morta è quello di Busòlo che, prima di andarsene, deposita sulle mani della defunta un garofano rosso.

11. Fogazzaro-Giacosa, n. 260 (Milano, 14 novembre 1901), pp. 320-321. Si veda anche Piero NARDI, «Disavventure teatrali del Fogazzaro», Pègaso. Rassegna di lettere e arti, luglio 1929 , pp. 40-47. 
«spunto drammatico» e "piccola tragedia comica». Ancora una lettera testimonia ulteriormente l'ammirazione incondizionata di Giacosa: «[...] Riletta stampata, la tua scena mi parve ancora più viva e poderosa e bella. [...] Quel breve dramma comico, a rileggerlo ed a ripensarlo è una cosa perfetta. Sono sicuro che lo si potrebbe recitare e sono sicurissimo che recitarlo avrebbe un successo trionfale. Già ti avevo altra volta domandato se non ti fosse mai venuto in mente di tentare il teatro. Questa volta, dopo questa prova, ti dico che sarebbe un peccato dei più gravi non farlo. Tu sì che saresti uno schietto rinnovatore della scena. [...] Provati amico mio. Anzi, poiché hai bell'e provato, buttati a nuoto addirittura». ${ }^{12}$ Fogazzaro sa bene che rischia di annegare nelle acque profonde della scena teatrale italiana se dovesse tentare una così ardua traversata! Nonostante tutto, l'insistenza e l'entusiasmo dell'amico muovono lo scrittore a tentare la prova delle scene, anche se rimane prudentemente scettico e chiede di optare per una prova teatrale anonima e di modificare il titolo come El garofolo de la siora Gegia. Giacosa approva e consiglia: "Credo fermamente che il Garofolo rosso sia cosa teatrale e ne garantisco un grande successo. La stessa cosa mi dissero persone di molto giudizio e di molta esperienza scenica». ${ }^{13}$ Inoltre, c'era già stata una proposta in merito da parte del conte Luigi Grabinski Broglio, direttore del teatro Manzoni di Milano, e pertanto Giacosa promette di contattare l'attrice Laura Zanon Paladini «che giudico la migliore attrice dialettale e la meglio adatta al caso. [...] Approvo l'idea dell'anonimo. Tutti sanno che la scena è tua, ma il N. N. vuol dire che non dai importanza a questo esperimento scenico». ${ }^{14}$ Ancora un'altra definizione: «esperimento scenico». Fogazzaro scriverà al Broglio per puntualizzare le proprie condizioni: «Egregio Signor Conte Le concederò dunque il mio Garofolo, però con la espressa condizione che non si faccia nessuna réclame e che il mio nome non figuri sul manifesto. È proprio una condizione sine qua non». ${ }^{15}$ La prudenza di Fogazzaro è dettata certamente dalla coscienza che un lavoro uscito di getto dalla sua fantasia, non confortato da alcuna esperienza in materia, potrebbe non reggere la prova del palcoscenico, come chiarisce, infatti, al fratello di Giuseppe Giacosa, Piero: «Il Garofolo fu scritto con grande rapidità e facilità [...]. Non pensavo che si potesse rappresentare. [...] L'esito è questo: che io concederò il Garofolo a patto che il mio nome non figuri nel manifesto, essendo quelle scenette troppo piccola cosa per affrontare con esse la prima volta, a viso aperto, il teatro. Pin [come era chiamato dagli amici Giuseppe Giacosa, N. d. C.] vuole che io scriva una commedia e io mi proverò ancora a immaginarne una come feci altre volte; ma non credo di riuscirvi». ${ }^{16}$ L'occasione per la rappresentazione delle «scenette» si presenterà un mese dopo. Ė sempre Giuseppe

12. Fogazzaro-Giacosa, n. 261 (Milano, 3 dicembre 1901), p. 322.

13. Ivi, n. 264 (Milano, 13 dicembre 1901), pp. 324-325.

14. Ibidem.

15. La lettera datata Vicenza, 15 dicembre 1901, è riprodotta in Fogazzaro-Giacosa, p. 325, nota 324

16. Lettere scelte, n. 589 (Vicenza, 15 dicembre 1901), p. 461. 
Giacosa a trovare l'aggancio. L'Associazione lombarda dei giornalisti, per recuperare fondi, aveva l'abitudine di promuovere degli spettacoli pubblici, e anche per quell'anno aveva deciso «di combinare una recita straordinaria alla quale concorreranno tutte le compagnie che sono oggi in Milano e che sarà il giorno 9 Febbraio (Domenica grassa). Capisci a che si arriva? Al Garofolo rosso. È qui la compagnia Zago e il tuo nome darebbe alla recita una inusata solennità e farebbe incassare un sacco di quattrini. Essi mi pregavano dunque perché intercedessi il tuo consenso». ${ }^{17}$ Fogazzaro approva sia l'idea (acconsentendo perfino che figuri il proprio nome), sia la compagnia teatrale, per quanto Giacosa sostenesse che l'attrice della compagnia Zago, Amalia Borisi, fosse meno adatta alla parte rispetto alla Zanon: «Carissimo. Se quei signori non esistevano, bisognava inventarli! Così va benone e io accetto qualunque attrice. Nel caso peggiore sarò, carissimo Caino, il tuo Abele». ${ }^{18}$ Lo scrittore allude, con sottile umorismo, al dramma lirico incompiuto di Giacosa, Caino, che in quei giorni sarebbe stato letto in pubblico da Francesco Pastonchi, al teatro Manzoni di Milano; ma la battuta è sintomatica: il sacrificio teatrale di Fogazzaro-Abele avrebbe avuto un sicuro carnefice in Giacosa-Caino. Alla fine l'altare su cui immolare El garofolo rosso è pronto; Fogazzaro farà in tempo a vedere la prova generale il giorno prima della recita (8 febbraio), insieme alla figlia Maria. ${ }^{19} \mathrm{Il}$ 9 febbraio 1902 El garofolo rosso fu portato sulle scene del teatro Manzoni nel corso della mattinata organizzata dall'Associazione lombarda dei giornalisti. Lo scrittore non assistette alla rappresentazione, ma fu messo subito al corrente dell'esito negativo, e ne dava immediato riscontro alla cugina Anna: «Un gran successo non è stato, perché il pubblico non gradì la tragica realtà dei cinismi e degli egoismi che si sfogano intorno al cadavere della contessa Marieta, e che io volli rappresentare perché caratteristici di quell'ambiente». ${ }^{20} \mathrm{La}$ critica è delusa e rimane, per rispetto dell'autore, in una ambigua vaghezza di giudizio tra la stroncatura e la «benevolenza». Ecco cosa scriveva Giovanni Vaccari sulla Rivista Teatrale Italiana, in una cronaca datata 11 febbraio 1902: «Il Fogazzaro, l'illustre romanziere idealista, ha voluto con questo "Garofano rosso" dare un tuffo in pieno verismo, scostandosi troppo dalle vie per cui egli è naturalmente chiamato [...]. Dall'arte possente e squisita del Fogazzaro potevamo attenderci, anche nel campo drammatico, qualcosa di più umano e più scenicamente fine e geniale». ${ }^{21}$

17. Fogazzaro-Giacosa, n. 272 (Milano, 27 gennaio 1902), p. 333.

18. Ivi, n. 273 (Vicenza, 28 gennaio 1902), p. 336.

19. «Io non vidi che la prova generale. Partii da Milano la sera prima della rappresentazione. Anche Maria venne meco e si divertì, alla prova, un mondo» (lettera ad Anna Fogazzaro, Vicenza, 13 febbraio 1902; cfr. LetTere scelte, n. 600, p. 468).

20. Ibidem.

21. Rivista Teatrale Italiana, Anno II, volume III, pp. 163-164. Si veda anche Nardi, p. 539, che riporta, tra l'altro, la testimonianza di Paolo Arcari, apparsa su Ateneo del 20 febbraio 1902: "poche proteste che avevano suono irriverente di fischi, furono soffocate da generale reazione di applausi, ché il momento sembrava disadatto alle lotte consuete». Dopo la prima, il lavoro fu replicato per tre sere al teatro dei Filodrammatici di Milano. 
Dopo l'esperienza del Garofolo rosso, anzi, durante questa esperienza (in alcuni momenti, le due vicende si sovrappongono), Fogazzaro lavora ad un altro bozzetto teatrale, certamente quasi pronto nel dicembre del 1901, come si ricava dalla citata lettera a Tommaso Gallarati Scotti: «[...] in questi ultimi giorni ho scritto un altro drammino Il ritratto mascherato chiestomi dalla Neue Freie Presse. È una eccezione che faccio alla regola di non dare a stranieri scritti inediti. Assediato, in passato, dalla N. F. P. mi compromisi con una mezza parola e adesso pagom. ${ }^{22}$ Giacosa, in una lettera del 7 gennaio 1902, fa un accenno al lavoro teatrale: "Piero mi scrive che hai pronto un altro lavoro scenico. È vero? E saresti disposto a dare anche di questo la primizia alla "Lettura"? Quale grande regalo mi faresti!».23 La risposta di Fogazzaro chiarisce meglio le ragioni del nuovo lavoro: «Per l'altro drammino (italiano e tutto serio) le cose stanno a questo modo. Ebbi in passato a sostenere un assedio della Neue Freie Presse per una novella. Me la cavai con l'infelice scappatoia di promettere per l'avvenire. Venne un mese fa il momento di tener parola. Scrissi allora il drammino ma feci le mie condizioni non volendo cedere un manoscritto originale all'estero, ciò che non feci mai e in ogni caso non farò più, per meno di quanto posso prendere in Italia. Non ebbi ancora risposta. Se la risposta o non viene o non mi soddisfa ti mando $\mathrm{Il}$ ritratto mascheratom. ${ }^{24}$ Giacosa non vedeva contrasti editoriali tra la possibile pubblicazione nella rivista viennese e la concomitante edizione italiana. ${ }^{25}$ Il 16 gennaio 1902, Fogazzaro avverte l'amico che gli ha "spedito testé il manoscritto»; e due giorni dopo Giacosa, ricevuto il testo, così commenta: "Carissimo. Il ritratto mascherato resterà fra le più belle cose tue. [...] Tu devi scrivere per il teatro. Vedrai all'esperimento della scena che questo secondo tuo atto è perfetto. Bisogna assolutamente farlo rappresentare. Io lo pubblicherò nel numero del

22. LetTere scelte, n. 591, cit., p. 463.

23. Fogazzaro-Giacosa, n. 266, p. 327.

24. Ivi, n. 267 (Vicenza, 10 gennaio 1902), p. 328; si veda anche LetTere sCelte, n. 613, pp. 478-479 (ma Gallarati Scotti, per errore, pone come data alla lettera il 10 maggio 1902; cfr. Marta Giacometti, Autografi ed edizioni dei carteggi fogazzariani, in Gilberto Pizzamiglio-Fabio Finotti (a cura di), Antonio Fogazzaro, tra storia, filologia e critica, Vicenza: Accademia Olimpica, 1999, p. 96, nota 18). A proposito della collaborazione tra Fogazzaro drammaturgo e la Neue Freie Presse riportano notizie sia Piero Nardi sia Tommaso Gallarati Scotti (Cfr. Nardi, pp. 539-540, e Gallarati Scotti, p. 355). Fogazzaro effettivamente inviò alla rivista copia del manoscritto, come testimonia la lettera a Gallarati Scotti del 31 gennaio 1902: "Vorrei trovare il tempo, a Milano, di leggerle Il Ritratto mascherato. Io non l'ho, avendone spedita una copia a Giacosa e l'altra alla Neue Freie Presses) (Lettere SCElte, n. 598, p. 467). Gallarati Scotti indica erroneamente il 20 marzo 1902, come data di stampa del Ritratto mascherato sulla rivista viennese; in realtà il testo teatrale Das Bild mit der Maske fu pubblicato sul n. 13505 del 30 marzo 1902. Precedentemente, sempre sulla Neue Freie Presse, nel numero 13425 del 9 gennaio 1902, era stata pubblicata la versione tedesca del Garofolo rosso (Die rothe Nelke).

25. "Appena ebbi la tua che mi parlava dell'impegno preso con Vienna, pensai di scriverti che la pubblicazione in tedesco, nulla poteva nuocere a quella in italiano» (Fogazzaro-Giacosa, n. 268, Milano, 14 gennaio 1902, p. 328). 
$1^{\circ}$ Marzo». ${ }^{26}$ La risposta non si fa attendere: «Sono ben lieto e orgoglioso del tuo giudizio, ma diffido sempre della mia attitudine a comporre una vera commedia. [...] È vero che Il Ritratto pare anche a me rappresentabile». ${ }^{27} \mathrm{E}$ certo dunque che sarà Giacosa a pubblicare per primo Il ritratto; una cartolina postale del 27 gennaio testimonia che Fogazzaro attende le bozze del dramma, che riceverà puntualmente tre giorni dopo. ${ }^{28} \mathrm{Nel}$ numero 3 del $1^{\circ}$ marzo 1902, Il ritratto mascherato appare sulle pagine della Lettura (pp. 193-200), sarà questa l'unica pubblicazione italiana del testo prima della definitiva raccolta dell'intera drammaturgia fogazzariana nel volume Scene, del $1903 .{ }^{29}$ È sempre Giacosa a prendere l'iniziativa per una possibile rappresentazione del lavoro: "[...] Mi dai licenza di scrivere fin d'ora alla Tina di Lorenzo per annunciarle la gran ventura? Perché credo in coscienza che la migliore attrice per la parte di Cecilia, sia quella. Dopo di lei potrai affidare quella parte all'Irma Gramatica». ${ }^{30}$ La possibile seconda opzione per l'attrice Irma Gramatica nasceva da un dato fisionomico legato alla protagonista Cecilia: «La tua lettera dice che Cecilia, deve essere pallida passionale, interessante e non bella. Ciò risponde al fisico dell'Irma Gramatica. Ma temo che questa non sappia esprimere con giusta misura, quella passione contenuta, quella signorile e passionale compostezza che è carattere del personaggio e che la parola rende a maraviglia». ${ }^{31}$ Nonostante i dubbi di Giacosa la scelta cadde sulla Compagnia Talli, di cui l'attrice Gramatica faceva parte. C'era solo un problema, la Compagnia Talli sarebbe rimasta a Venezia non oltre il 4 marzo, pertanto Giacosa cercava di affrettare i tempi: «Bisognerebbe quindi che il tuo atto andasse in scena non più tardi del $1^{\circ}$ dello stesso mese. [...] Del resto Venezia, specialmente in stagione teatrale di soli 20 giorni, non è città da molte repliche. $\mathrm{E}$ andando in scena il giorno 1 si potrebbero sempre fare tre rappresentazioni. Che se il giornale di Vienna non ci si oppone, si potrebbe dare la prima recita il 28 Febbraio, giorno di Venerdì, che è appunto la giornata preferita per le grandi novità».32 La "Neue Freie Presse» non darà alcun parere sfavorevole, e dunque Giacosa si ritiene libero di definire meglio i contatti con la Compagnia Talli: «Io scrivo una lunga lettera all'Irma Gramatica, cercando di dirle, nel suo linguaggio, come a mio vedere vada intesa la parte di Cecilia. In pari tempo scrivo a Talli per la distribuzione delle parti che è cosa importante. Tu faresti bene ad assistere ad una o due prove. Non, se mi dai retta, alle primissime e non all'ultima. Anzi all'ultima, non conviene, data la tua autorità,

26. Fogazzaro-Giacosa, n. 270, p. 330. Si veda anche Piero Nardi, Fogazzaro su documenti inediti, Vicenza: Jacchìa, 1929, p. 80.

27. Ivi, n. 271, cit.

28. «Carissimo. Ieri mattina ricevetti per espresso le bozze del Ritratto da restituire al più presto; e infatti ripartono col diretto delle 10» (Fogazzaro-Giacosa, n. 276, Vicenza, 31 gennaio 1902, p. 338; ma si veda anche n. 275, pp. 337-378).

29. Si tratta del volume Antonio Fogazzaro, Scene, Milano: Baldini e Castoldi, 1903.

30. Fogazzaro-Giacosa, n. 270, cit., pp. 330-331.

31. Ivi, n. 272 (Milano, 27 gennaio 1902), p. 335.

32. Ivi, n. 281 (Milano, 17 febbraio 1902), p. 342. 
e trattandosi di un primo esperimento, non conviene che tu assista, per non mettere in soggezione gli attori che già quel giorno saranno eccitati e conturbati». ${ }^{33}$ Fogazzaro sarà a Venezia il 22 febbraio: «Sono qui [...] per il Ritratto che lessi oggi agli attori». ${ }^{34}$

La rappresentazione ebbe luogo il 26 febbraio al teatro Goldoni, interpretata da Irma Gramatica, Laura Vestri Marsoni, Ruggero Ruggeri, e fu un clamoroso fiasco. Fallimento causato in parte da un'esecuzione frettolosa e di bassa qualità, in parte dall'incapacità del pubblico di accettare lo «spirito del lavoro»: dalla platea colpi di tosse, proteste e fischi, contro un unico applauso (contestato anche questo). ${ }^{35}$ Stupore da parte di Giacosa: «Come può essere che il tuo breve rapido ed intimo dramma, non sia piaciuto al pubblico di Venezia? Già mi sarebbe parso inesplicabile il silenzio, ma le disapprovazioni sconvolgono addirittura tutte le idee che mi sono venuto formando sul teatro. Già sono persuaso che la rappresentazione fu troppo precipitosa». ${ }^{36}$ Le prove furono effettivamente poche (cinque) e, secondo la testimonianza di alcuni, gli attori erano impreparati, come conferma anche Giacosa: «Ieri vidi il Fradeletto [noto critico teatrale, N. d. C] e si parlò del Ritratto. Mi disse che nessuno sapeva la parte, ma devo pur riconoscere che nemmeno lui non ha capito. Mi parli di ritentare la prova. A Parma no certo, e direi, con quella compagnia in nessun

33. Ivi, n. 282 (Milano, 21 febbraio 1902), p. 344.

34. Lettera a Tommaso Gallarati Scotti (Venezia, 22 febbraio 1902); cfr. Lettere scelte n. 601, pp. 468-469. Nel post scriptum Fogazzaro dichiara: «Se il Ritratto farà fiasco, cosa ben possibile, credo che me ne consolerò facilmente nella coscienza di non averlo meritato».

35. Due giorni dopo l'insuccesso Fogazzaro scriveva a Giacosa (Vicenza, 28 febbraio 1902) facendo il resoconto dell'incontro e delle prove con gli attori: «Andai a Venezia Sabato dopo aver telegrafato al Talli che venne all'albergo con la Gramatica e il Calabresi. Lessi la commedia lì, all'albergo. Fu ascoltata con attenzione molto viva ma, finita la lettura, capii subito che l'impressione non era stata troppo favorevole. [...] L'indomani mi recai in teatro per assistere all' impostazione, come mi dissero. Questa lettura, con la parte in mano, nel complesso non mi lasciò malcontento. Notai però negligenze che a me novizio parevano strane. La Trechi neppur sapeva di che si trattasse, credeva alla sincerità della sua storia! Il domestico mi parve pessimo [...] Il Talli [...] aveva riconosciuto, il Sabato, che la commedia era difficile a recitare appunto per gli accenti, le sfumature, le pause ecc... Ma insomma Domenica egli e la Gram. [atica] consentivano che si poteva perfettamente andare in scena prima di venerdì [...] Mercoledì ritornai a Venezia, con Maria. Mi si disse ch'erano state fatte quattro prove. Assistetti all'ultima. [...] A ogni modo lasciai il teatro colla persuasione che malgrado una esecuzione non perfetta, il $R$. avrebbe dovuto commuovere, piacere! [...] Ecco tutta la triste storia. [...] Ecco delle sensazioni nuove che ho attraversate. Forse, alla mia età, non era proprio necessario» (Fogazzaro-Giacosa, n. 286, pp. 349-353). Cfr. anche Lettere scelte, n. 603, pp. 470-472 e Gallarati Scotti, pp. 358-360.

36. Fogazzaro-Giacosa, n. 284 (Milano, 27 febbraio 1902), pp. 346-347. I giornali tentarono di attenuare lo smacco, ma furono comunque fortemente critici: «ll Fogazzaro ha avuto con questo suo lavoruccio in un atto una buona intenzione, quella di manifestare sulla scena il suo idealismo religioso. Il male è che se l'opera compiuta può ancora dirsi una buona intenzione, deve giudicarsi una nullità artistica. Questo povero atto è così insignificante e puerile $[\ldots]$ da non poter essere neppur compatito come il lavoro di un principiante. [...] Gli attori non hanno fatto gran che per sostenere il lavoro e del resto poco c'era da fare» (Gazzetta di Venezia, 27 febbraio 1902). 
luogo. Io proverei ora colla Tina Di Lorenzo». ${ }^{37}$ Pronta risposta di Fogazzaro: "Quanto al Ritratto, io, a mente riposata, mi domando chi lo capirà in teatro se Fradeletto non lo ha capito e dubito della opportunità di riprodurlo»; e, qualche riga dopo: «io lo pubblico da Baldini e Castoldi che me l'hanno chiesto con il Garofolon. ${ }^{38}$ Intanto, sempre a fine febbraio, un nuovo allestimento del Garofolo veniva presentato a Padova, sempre dalla compagnia Zago, e, successivamente, nell'autunno del 1902, al teatro Rossini di Venezia, con scarso successo in entrambi i casi, tanto che Fogazzaro decide di rinunciare ad ulteriori tentativi. ${ }^{39}$ Lo stesso accadeva per il Ritratto, che vedeva Fogazzaro ormai rinunciatario: "Quanto al Ritratto mascherato i miei desideri di rivincita sono molto molto sbolliti e quasi preferirei non tentare più la sorte». ${ }^{40}$ L'avventura sulle scene italiane dello scrittore vicentino era ormai giunta alla conclusione. Forse, alla fine, Abele era stato davvero sacrificato da Caino...

Nonostante gli insuccessi, ancora una volta si metterà alla prova con un altro testo teatrale, il terzo e ultimo di quel febbrile anno. Si tratta di Nadejde, ${ }^{41}$ il testo più complesso ed articolato nella struttura diegetica, che risultava, pertanto, ancor meno rappresentabile sulle scene: "A parte difficoltà estrinseche per la rappresentazione, come intervalli di ore tra episodio ed episodio del secondo atto; a parte certi soliloqui esplicativi, contrari a ogni principio di verosimiglianza e a ogni buona norma teatrale moderna, non so se Nadejde avrebbe avuto sul palcoscenico miglior fortuna del Garofolo rosso e del Ritratto mascherato». ${ }^{42}$ Il testo doveva essere già scritto nel luglio del 1902, come si

37. Ivi, n. 288 (Milano, 3 marzo 1902), pp. 354-355.

38. Ivi, n. 289 (Vicenza, 8 marzo 1902), p. 356.

39. "Quanto al Garofolo rosso lasciamolo appassire in pace» (MANGinI, p. 221).

40. Fogazzaro-Giacosa, n. 292 (Vicenza, 14 aprile 1902), p. 359.

41. La vicenda di Nadejde è questa: la principessa russa Tatiana, separata dal principe de La Roche, vive con la figlia di entrambi, la diciottenne Nadejde, in una lussuosa villa sulla riva del lago di Como, ed è sul punto di ricevere la visita dei Granduchi della Russia, Ivan e sua moglie. La situazione è complicata, da una parte, perché Tatiana e Ivan sono amanti, e dall'altra perché l'etichetta esige che durante l'illustre visita la principessa Tatiana sia accompagnata dal marito. Quindi, Tatiana si è dovuta piegare alle esigenze economiche del marito, che pretende una grossa somma di danaro per essere presente. Nadejde, che adora suo padre, e nonostante sua madre le riveli che egli venga alla villa spinto soltanto da un interesse puramente economico, è ansiosa di rivederlo dopo dieci anni di lontananza. Nella seconda parte, mentre Nadejde aspetta sola il momento dell'incontro con suo padre, sente le voci di alcune persone che alludono alla dissoluta vita di sua madre, che, però, la ragazza ritiene calunniose. Avviene l'incontro, e Nadejde implora al padre di cambiare vita, ma invano; costui le promette di tornare più tardi a rivederla, quando i Granduchi saranno partiti. Nadejde attende nei pressi dell'uliveto. Nel frattempo, Tatiana e il Granduca Ivan si sono dati un appuntamento segreto, e Tatiana consegna all'amante una chiave con cui aprire il cancello dell'imbarcadero: Ivan prova la chiave e lascia il cancello aperto. I due amanti si appartano nell'uliveto della villa, e Nadejde, involontariamente, sarà testimone del loro segreto amore. Sconvolta, la ragazza rimane sola e triste, e, poco dopo, verrà a sapere che suo padre, ubriaco fradicio, è già partito scordandosi di lei. Allora Nadejde, trovando il cancello dello sbarco aperto, rassegnata, si annega nelle acque del lago.

42. NARDI, p. 543. 
ricava da una lettera a Tommaso Gallarati Scotti: «Fra l'altro ho finito un drammino, scritto per una delle mie solite deboli compiacenze, non da rappresentare e intitolato Nadejde». ${ }^{43}$ Ed infatti, il dramma rimase sulla carta e mai fu rappresentato; qualche mese dopo fu pubblicato sull' "Almanacco italiano» (1903), ed infine compreso nel volume Scene, insieme al Garofolo rosso e al Ritratto mascherato. ${ }^{44}$ In Scene ogni testo teatrale è preceduto da una dedica introduttiva curiosamente indirizzata ad un protagonista di ogni singolo dramma. La prima è rivolta "All'eccellentissimo signore Checco Busòlo» (il cinico marito della protagonista del Garofolo rosso); la seconda «Alla signora Bice Mannelli» (la madre di Cecilia del Ritratto mascherato); la terza "A suor Paula Ritter» (istitutrice di Nadejde). ${ }^{45}$ Sul Corriere della Sera (16 settembre 1903) Giacosa aveva recensito positivamente il volume Scene, e in questa occasione l'amico «Pin» si era accollato pubblicamente la responsabilità degli insuccessi sul palcoscenico dei due drammi dello scrittore vicentino, il quale bene-

43. Lettere scelte, n. 617 (Tonezza, 24 luglio 1902), p. 482. Ma ecco cosa scriveva a proposito di Nadejde Yole Moschini: «Le ho rispedito ieri stesso le bozze [...]. Le lessi con tutto l'interesse che derivava dall'essere un lavoro Suo e dalla fidente gentilezza da Lei mostratami. Come al solito, nelle brevi scene si sono constipate idee, intenzioni, osservazioni profonde, fini, con sprazzi di luce che permettono a chiaroveggenti di penetrare lo sguardo in vite umane intere [...]. Ma se ad apprezzare ciò che risulta da quei dialoghi, forse un poco artificiosi, ci vorrebbero lettori quasi già iniziati al genere, quali spettatori si dovrebbero esigere? Io spero che questa volta Ella non cederà alle pressioni di chi volesse far rappresentare il piccolo grande dramma" (Fogazzaro-Moschini, n. 238, Misurina, 7 agosto 1902, p. 369). Si veda anche NARDI, pp. 543-544.

44. La pubblicazione sull' Almanacco Italiano fu quasi analoga a uno smacco sulle scene. Fogazzaro si era deciso a concedere il suo nuovo lavoro all'editore Bemporad, su pressioni dell'amico Pompeo Molmenti, ma non aveva considerato il carattere commerciale della rivista, straripante di pubblicità a piena pagina. Il testo apparve pertanto nel volume VIII del 1903 (ma edito entro il dicembre del 1902) inframmezzato di annunci economici: «Se vedi il signor Bemporad, digli che non mi mandò ancora una sola copia dell'Almanacco. Sei liberissimo di tartassare per quel miscuglio di réclame, il Bemporad e me che non mi premunii» (lettera a Piero Giacosa, Vicenza, 26 dicembre 1902); e ancora, parlando con Filippo Crispolti della prossima pubblicazione di Nadejde su Scene, si lamentava: «quando mai cedetti al Molmenti che me la fece dare al Bemporad per poi venire conciata così!» (Vicenza, 14 gennaio 1903); cfr. LetTere scelte, pp. 496-497 e p. 502. Una curiosa attestazione di questo "fattaccio» editoriale è riportata da Domenico Giuriati nel suo saggio Il plagio. Furti letterari, artistici e musicali (Milano: Hoepli, 1903): "Come la industria mercantile sappia speculare sull'arte, mente umana non arriva a immaginare. Leggo e ammiro Nadejde, azione drammatica in due parti, di Antonio Fogazzaro, vale a dire di uno fra gli spiriti più alti e de' caratteri più intemerati che fioriscano nella patria letteratura. La leggo in un Almanacco di quest'anno, e fin qui pazienza! Anche gli almanacchi hanno il diritto di ornarsi e di azzimarsi; ma ciascuna pagina del testo è incastrata fra due pagine di avvisi a pagamento, per modo che l'attenzione del lettore è necessariamente non sviata, costretta a divagare nella farina lattea, nel Sapol, nelle tinture, nelle tossi, nei catarri, nel verme solitario - e peggio!» (pp. 478-479).

45. "Ho scritto di questi giorni la dedica del Garofolo rosso al signor Checco Busòlo e la dedica al Ritratto mascherato, alla signora Mannelli madre di Cecilia. Mi pare non sieno riuscite male. A Roma scriverò la terza dedicata alla signorina Paola Ritter» (lettera a Tommaso Gallarati Scotti, Vicenza, 24 febbraio 1903; cfr. Lettere scelte, n. 644, p. 505). 
volmente rispondeva: «Sei stato regalmente generoso! Non so se l'abnegazione con la quale rivendichi a te la responsabilità dei miei fiaschi teatrali basterà $\mathrm{a}$ farmi assolvere. Io stesso mi dico che avrei dovuto resistere. Soggiungo però subito che se ho ceduto con qualche fatica per il Garofolo, non è stato così per Il Ritratto nel successo del quale avevo fede e ch'era invece destinato a maggior rovina». ${ }^{46}$ Chiudiamo la cronaca di questa breve vicenda teatrale, riportando la spiritosa risposta di Fogazzaro a Vittoria Aganoor, che gli riferiva il giudizio entusiasta su Nadejde espresso dall'amico Giacosa (che la poetessa definisce «un ammiratore cocente» dell’opera di Fogazzaro): «Sì, il mio carissimo Giacosa mi vuole un gran bene e colla sua ammirazione per le mie opere drammatiche mi farebbe fischiare fino alla mia ultima e definitiva scomparsa dalla scena del mondo». ${ }^{47}$

\section{Il Ritratto mascherato}

La vicenda del dramma è semplice; l'azione avviene tutta nella stanza-studio del defunto Carlo Festi.

Cecilia, vedova - il marito, un noto scrittore, è deceduto qualche giorno prima -, si trova nello studio di quest' ultimo in attesa dell'arrivo del notaio e di alcuni parenti per il recupero del testamento custodito in un cassetto della scrivania. Mentre attende nel dolore e nella rispettosa memoria del marito, irrompe nella stanza, trafelata, la signora Trechi, moglie del notaio incaricato dell'esecuzione testamentaria. Costei racconta una strana vicenda di debiti contratti con il marito di Cecilia per aiutare alcune persone a lei care e in difficoltà economiche. Chiede di poter aprire il fatidico cassetto per recuperare, prima che il notaio arrivi e se ne accorga, un'obbligazione contratta e ormai saldata con il defunto marito di Cecilia. Quest'ultima, non avendo la chiave, non può accontentarla; promette, però, di far sì che il documento non venga scoperto. La donna esce, e subito dopo arrivano il cognato di Cecilia e il padre di lei, ed infine il notaio. Il cognato, che ha incontrato poco prima la signora Trechi, che anche a lui ha fatto identiche richieste sulla presunta obbligazione, cerca di non far aprire il cassetto, adducendo come scusa la mancanza della chiave a lui affidata ma dimenticata. Tutto sembra risolto, sennonché il notaio, con una propria chiave, riesce a forzare la serratura. Confusione e tentativi di impedire che quest'ultimo metta mani nel cassetto. Alla fine il testamento è trovato, senza che siano scoperte altre carte, e i tre uomini possono andare dal Pretore per la lettura ufficiale. Cecilia rimane in casa con la madre. Ora può finalmente mettere ordine tra le carte del marito e cercare la famosa quietanza del prestito. Tra le carte, però, saltano fuori alcune lettere

46. Fogazzaro-Giacosa, n. 299 (Valsolda, 17 settembre 1903), pp. 364-366. Cfr. anche LetTere SCELTE, n. 656, p. 516.

47. Lettera da Vicenza del 2 febbraio 1903, pubblicata in Adriana Chemello e Donatella Aless (a cura di), Tre donne d'eccezione: Vittoria Aganoor, Silvia Albertoni Tagliavini, Sofia Bisi Albini, dai carteggi inediti con Antonio Fogazzaro, Padova: Il Poligrafo, 2005, p. 132. Si veda anche Fogazzaro-Giacosa, p. 365, nota 372. 
e la fotografia di una donna sorridente, in toilette da ballo e con il viso coperto da una maschera. Se qualche dubbio sullo strano comportamento della signora Trechi, del cognato e del padre aveva insospettito Cecilia, ora la scoperta del ritratto e l'insistenza della madre, che si affanna a cercare giustificazioni, non richieste, per quella foto e quelle lettere, non lasciano spazio ad altre verità. Cecilia è ora cosciente del probabile tradimento del marito, ma il suo amore idealizzato, la consacrazione che ha di lui non le permettono di sporcare con un dubbio simile la memoria del defunto. Il suo amore assoluto la condurrà al gesto finale: quello di bruciare il ritratto e le lettere nel fuoco del camino.

In questo breve atto unico, Fogazzaro fa uso di una serie di elementi narrativi caratteristici della propria esperienza di romanziere, incrociandoli con altri elementi tipici del teatro di fine secolo, ben collaudati e diffusi. L'elemento cardine della storia è l'amore ideale e assoluto di Cecilia, inattaccabile e fedele, disponibile al perdono, fino al sacrificio. Nella dedica, posta come prefazione al dramma, lo scrittore rimanda a un episodio realmente vissuto (l'incontro con la donna che ha ispirato la figura di Cecilia); lo spunto per il fatto narrato nasceva da una riflessione di carattere religioso incentrata sul principio cristiano del perdono, inteso come perdono divino, «intero», che rimette totalmente la pena e annienta l'offesa e «rimbianca» la memoria e l'anima del reo: "soggiunsi che perdonare così era degno di un amore onnipotente e che l'amore umano, il femminile sopra tutti, era qualche volta portato [...] a negar la offesa più flagrante o a giustificarla, a deificare l'amato, a odiare chi gli apponesse una imperfezione, una colpa». ${ }^{48}$ Questa è la forza interiore che, nel dramma, spinge Cecilia a reagire contro ogni sospetto, contro ogni allusione, contro ogni accusa presunta nei confronti del defunto marito. Antonio Stefani riconosce, in nuce, in questo dramma, la trama della commedia pirandelliana Tutto per bene. ${ }^{49}$ Solo che in quella, il protagonista Martino Lori risulta ingenuamente incosciente (incoscienza portata fino all'estrema «imbecillità», riconosciuta dallo stesso protagonista) dell'infedeltà della defunta moglie; qui Cecilia, consapevole ormai del tradimento del marito (che collima con le allusioni del padre e della madre), si oppone all'idea che la memoria del consorte e del loro amore venga infangata: all'ingenuità contrappone una ostinazione cieca. ${ }^{50}$ Sempre Antonio Stefani, inoltre, ha individuato alcuni elemen-

48. Osserva Luciano Morbiato: «l'argomento della conversazione, che passa insensibilmente dalla religione del perdono all'adulterio imperdonabile, appartiene alla soggetistica fogazzariana, nella quale l'idealizzazione della donna è tale che il dialogo d'amore equivale a una sacra conversazione. Lungi dall'essere confusa con i simboli della perdizione della fine secolo romantico-decadente [...], essa esercita la sua funzione salvifica, come una nuova Beatrice» (Fogazzaro-Starbuck, p. 108-109).

49. Si veda Stefani, p. 23. La commedia di Pirandello, tratta da una novella del 1906, fu portata sulle scene nel 1920 .

50. In altra sede abbiamo alluso al nomen omen di Cecilia. Questo nome ricorre altre volte nell'opera di Fogazzaro: dà il titolo ad una poesia della raccolta Valsolda (1876), ma soprattutto è il nome dell'antenata di Marina in Malombra, di cui crederà d'essere la reincarnazio- 
ti strutturali nell'opera drammaturgica dello scrittore vicentino, che "presi assieme, dimostrano di essere collegati a ben guardare da un fil rouge non proprio casuale». ${ }^{51}$ Elementi che si ritrovano in tutti e tre i drammi, ma che nel Ritratto mascherato risultano paradigmatici. Uno di questi è quello "della crisi, della disgregazione della coppia nei confronti del contratto matrimoniale», motivo fondamentale di molta letteratura fogazzariana. ${ }^{52}$ Cecilia è una donna fedele al proprio uomo, fedele all'istituzione matrimoniale, perfino sottomessa per la propria «ignoranza» al marito «intellettuale», 53 ma il tradimento (tema fin troppo abusato dal teatro borghese e dalla letteratura della seconda metà dell'Ottocento) è sempre in agguato, e anche Cecilia deve fare i conti con la drammatica scissione degli affetti e del dovere coniugale. Fogazzaro appena un anno prima aveva terminato e pubblicato Piccolo mondo moderno (1901), dove il tema dell'infedeltà coniugale e dell'adulterio è il movente che determina la gran parte dei tormenti interiori del giovane Piero Maironi. A guardar bene, la forza di volontà e di sacrificio, la fedeltà di Cecilia sono esattamente la proiezione della fedeltà assoluta che Elisa, la moglie «demente» di Piero (ma ben cosciente della relazione tra quest'ultimo e Jeanne Dessalle), saprà ben esprimere poco prima di morire, in un ultimo momento di lucidità: «Non sono stata una buona moglie - zitto caro, zitto - no, ti volevo tanto bene, tanto tanto e non ho saputo dimostrarlo, devi avermi creduta fredda, è stato un gran male, adesso lo capisco. [...] Caro, vuoi che ci perdoniamo tutto? Proprio tutto tutto? Anche quello che tu non sai di me? Anche quello che io non so di te?». ${ }^{54}$ Cecilia risponde così a quell'ideale femminile, forte e integerrimo, anche nel dolore, che aveva visto in Elena del Daniele Cortis la sua massima espressione: ${ }^{55}$ "Cecilia Festi è composta della sostanza delle donne amanti del Fogazzaro. [...] una creatura profondamente vera, [...] donna di vita interiore, apparentemente comune, fredda, non colta, ma con un mondo

ne («Cambiati nome! Che io torni a essere Cecilia»); cfr. Roberto RandaCcio, «Trattando l'ombre come cosa salda. Considerazioni sull'onomastica nell'opera di Antonio Fogazzaro", RION. Rivista Italiana di Onomastica, xIV (2008), 1, pp. 89-108.

51. Stefani, p. 19. A questi tratti comuni possiamo aggiungere quello, che si dà quasi per scontato in tutta l'opera fogazzariana, ossia la rappresentazione di un ceto medio-alto della società italiana, con ville aristocratiche, personaggi altolocati e serventi fedeli, come confermato anche da Morbiato: «per Fogazzaro la collocazione sociale della donna è importante, non può bastare l'elevatezza intima, morale, deve esserci anche quella sociale» (FogAzZAroStarbuck, p. 109).

52. Stefani, p. 19.

53. Non possiamo non leggere tra le righe un'allusione autobiografica, pur minima, riferita allo scrittore vicentino e al suo rapporto "contrastato» con la moglie, relegata molto spesso a un ruolo secondario nelle vicende letterarie del marito, ma in una subordinazione presente e vigile sulle avventure intellettuali pubbliche e private del marito.

54. Antonio Fogazzaro, Piccolo mondo moderno, a cura di Roberto Randaccio, introduzione di Daniela Marcheschi, Venezia: Marsilio, 2012, p. 348.

55. Alcune eroine di Fogazzaro hanno un passato coniugale turbato da esperienze negative fatte di abusi e soprusi, con mariti violenti e alcolizzati, dissoluti, corrotti e scialacquatori; accade a Elena, a Jeanne Dessalle, perfino all'egocentrica Tatiana del dramma Nadejde. 
chiuso di sentimenti eccezionali, con una potenza di grandezza eroica non compresa del mondo profano: una delle tante a cui forse passiamo vicino con indifferenza; ma in cui il Fogazzaro sprofonda lo sguardo e vede un dramma». ${ }^{56}$

Altro elemento comune ai tre drammi è la Morte; è questo un elemento cardine di quasi tutta l'opera di Fogazzaro romanziere. Il momento drammatico della morte è fenomeno che determina lo sbocco finale di una tragedia interiore (Malombra); conduce al mutamento esistenziale, psicologico e morale (Piccolo mondo moderno); diviene il simbolo di nuova vita, di rinascita morale e religiosa e di speranza futura (Piccolo mondo antico, Piccolo mondo moderno, Il Santo). Nel Garofolo rosso la morte è conseguenza dell'egoismo e della cattiveria; in Nadejde è atto finale che scaturisce in un gesto estremo di sacrificio $;^{57}$ nel Ritratto mascherato la morte è già arrivata, ma aleggia sulla scena come una presenza evocata, come il persistente suono nell'aria di una romanza mai dimenticata («Sai, mamma, che l'ho chiamata io la morte?»). Nel dramma vale il principio cristiano del parce sepulto, inteso come il rispetto del morto, che attraverso il perdono e le preghiere dei vivi può «sbiancare» la propria anima e ritrovarsi al cospetto di Dio privo di peccati, super nivem, e non il concetto borghese del «mettere una pietra sopra», dell'insabbiare lo scandalo, del nascondere sotto una finta, apparente normalità il malessere familiare ( $(\mathrm{E} \mathrm{tu}, \mathrm{mamma}$, mi vieni fuori, adesso, con questi miserabili sospetti, con queste offese! Sì, sì, tu hai paura che io scopra chi sa cosa!»).

Infine, un piccolo deus ex machina per entrambi i drammi: una piccola chiave. ${ }^{58}$ Quella chiave che, nel Ritratto mascherato, apre il cassetto tabuato e rivela, attraverso la fotografia della bella donna dal volto mascherato, un segreto, un «mistero» ripetendo ancora una volta la collaudata meccanica scenica di molte opere dello scrittore: la scoperta di un documento segreto che deter-

56. Gallarati Scotti, pp. 355-357.

57. La "morte per acqua» è un altro importante elemento dell'architettura romanzesca di Fogazzaro. Marina, in Malombra, scomparirà tra le acque del lago; Maria/Ombretta, in Piccolo mondo antico, morirà affogata sempre nelle acque del lago (e una fine simile rischiò di farla lo sfortunato figlio dello scrittore, Mariano, all'età di dodici anni); sempre nello stesso romanzo, sua madre Luisa, disperata, è tentata dal suicidio addentrandosi nelle acque del lago. Ma ricordiamo anche la protagonista di Leila, che si distende seminuda, di notte, nell'acqua fredda del ruscello, come una Ofelia preraffaellita penitente.

58. Rimandiamo per un più esaustivo chiarimento dei singoli episodi a STEFAnI, pp. 19-30. L'episodio della chiave del Ritratto mascherato aveva comunque originato una breve discussione scenica tra Giacosa e Fogazzaro, anche questa testimoniata dal loro fitto epistolario. Giacosa osservava che: «dopo che il notaio ha aperto il cassetto, io non tornerei più sulla chiave. Già quello là che se la leva di tasca per darla a Cecilia non mi par naturale. E poi perché ridargliela quando il cassetto può esser lasciato aperto? E se anche la cosa fosse vera, la leverei perché ha l'aria di un piccolo artificio per sospendere una volta di più l'attenzione». Risponde Fogazzaro: «Allora parleremo della chiave. Vero che va un po' in lungo ma è anche vero questo: il cognato e il padre, sapendo quello che sanno, devono necessariamente pregare il notaio di chiudere; Cecilia necessariamente desidera che resti aperto e di fronte all'espresso suo desiderio mi parve inevitabile che il notaio offrisse la chiave. Se avessi potuto abbreviare avrei abbreviato volontieri, ma quella necessità che qui sottolineai è assoluta»; cfr. FogazzAro-Giacosa, n. 272, cit., e n. 273, cit. 
mina un cambiamento radicale nella vicenda. Accade con il manoscritto dell'antenata Cecilia nascosta nello stipo segreto in Malombra, accade con le carte occultate dell'eredità Maironi in Piccolo mondo antico, accade con il biglietto dalla drammatica scritta "S'ofro» in Piccolo mondo moderno; scoperte che determineranno nelle vicende dei personaggi mutamenti sostanziali, che sfoceranno nel dramma o nel sacrificio. ${ }^{59}$ Qui, al contrario, Cecilia vive il suo dramma senza volerlo comprovare, nel rispetto della memoria del marito e nella ostinata accettazione di un perdono assoluto che non può permettere profanazioni di nessun genere. Il fuoco purificatore renderà giustizia di tutte le allusioni, di tutti i sospetti, di tutte le impudenti dicerie. Cecilia ci appare così un personaggio originalissimo per le scene, fortemente idealizzato, ma, al contempo, disarmante nella sua strenua e onesta difesa del proprio amore. Il pubblico non capì la novità del soggetto e la forza interiore della protagonista: «Forse in certi ambienti non si conoscono donne come Cecilia! Quando io lessi il $R$. [itratto] a Ina Franco, finita la lettura esclamò: «ah come è vera!», perché è appunto una delle Cecilie, rarissime, che pure il mondo ha. Altro che Cecilia non era da cercare nella commedia; ossia, era a cercarvi quella immagine della realtà che a me pare di trovarvi». ${ }^{60}$

\section{Bibliografia}

Fogazzaro-Giacosa

Antonio Fogazzaro-Giuseppe Giacosa, Carteggio (1883-1904), a cura di Oreste Palmiero, Vicenza: Accademia Olimpica, 2010.

FogaZZARO-MOSCHINI

Antonio Fogazzaro-Yole Biaggini Moschini, Carteggio (1887-1909), a cura di Viviana Bertoldo e Piero Luxardo, Vicenza: Accademia Olimpica, 2011.

FogazZARO-STARBUCK

Antonio Fogazzaro-Ellen Starbuck, Carteggio (1885-1910), a cura di Luciano MorBIATO, Vicenza: Accademia Olimpica, 2000.

Gallarati Scotti

Tommaso Gallarati Scotti, Vita di Antonio Fogazzaro, Milano: Mondadori, 1963, $2^{\mathrm{a}}$ ed. (1 ${ }^{\mathrm{a}}$ ed. 1934).

LETTERE SCELTE

Antonio Fogazzaro, Lettere scelte (1860-1911), a cura di Tommaso Gallarati SCOTTI, Milano: Mondadori, 1940.

59. Come osserva anche Floriano Romboli nella sua indagine sui racconti di Fogazzaro: «mi pare che proprio i temi del sacrificio e del mistero costituiscano i nuclei aggreganti la materia dei racconti, che in forza di questi acquistano coesione strutturale e complessivo, rigoroso significato, al di là degli occasionali spunti inventivi» (Antonio Fogazzaro, Racconti, a cura di Floriano Romboli, Milano: Mursia, 1992, pp. 16-17).

60. Fogazzaro-Giacosa, n. 286, cit., p. 352, e Lettere scelte, n. 603, cit., p. 471. E, quasi a difendere le proprie posizioni e le proprie teorie contro il giudizio dei critici, lo scrittore chiarisce all'amico Giacosa che "la prefazione del Ritratto fu scritta perché... diciamolo, per dispetto» (Fogazzaro-Giacosa, n. 299, cit., e Lettere scelte, n. 656, p. 516). 


\section{MANGINI}

Nicola MAngini, Il teatro veneto moderno. 1870-1970, Roma: Istituto della Enciclopedia italiana, 1992.

NARDi

Piero Nardi, Antonio Fogazzaro, Milano: Mondadori, 1945, $3^{\text {a }}$ ed. (1 $1^{\mathrm{a}}$ ed. 1938).

\section{STEFAni}

Antonio Stefani, «Una chiave per il teatro di Antonio Fogazzaro», Vicenza: Club Amici del Teatro di Vicenza, 1984; ora riproposto, suddiviso in due parti e con qualche lieve modifica, in Fitainforma, XXV, n. 2 (giugno 2011), pp. 25-27, e n. 3 (ottobre 2011), pp. 25-27.

\section{Criteri dell'edizione e della traduzione}

Per la fissazione del testo abbiamo scelto come edizione di riferimento quella compresa nel volume Scene, Milano: Baldini \& Castoldi, 1903, pp. 61-122 (SCENE 1903), che abbiamo rispettato anche nei criteri tipografici. Per soluzioni puntuali, che si indicano in nota, abbiamo tenuto anche conto di quella precedente de La Lettura, Marzo 1902, pp. 192-200 (LeTTURA I9O2) e di quella di Tutte le opere di Antonio Fogazzaro a cura di Piero Nard, Milano: Mondadori, 1944, vol. Xv, Scene e prose varie, pp. 35-63 (NARDI I944). Nel testo lo scrittore riproduce alcuni versi tratti della romanza di Robert Schumann, Frauenliebe und leben (op. 42, del 1840), su testo di Adalbert von Chamisso, che Fogazzaro ripropone nella versione italiana (Amor di donna) fatta da Vittorio Radicati di Marmorito. ${ }^{61}$

Com'è stato detto nell'introduzione, l'edizione in Scene era preceduta da una dedica, che qui riproduciamo tradotta in spagnolo, mentre l'originale italiano è in nota. ${ }^{62}$

61. Si veda Fogazzaro-Giacosa, n. 269 (Vicenza 16 gennaio 1902), pp. 329-330, e LetTERE SCELTE, n. 593, pp. 464-465. In una nota posta in fine testo del Ritratto mascherato pubblicato su La Lettura, si dice chiaramente: «I versi di Chamisso, musicati da Roberto Schumann, son riferiti nella traduzione, assai efficace, del conte Vittorio di Marmorito». La predilezione dello scrittore per l'opera di Schumann è testimoniata dalle numerose citazioni presenti nelle sue opere, a partire dal riferimento all'op. 68 nell' intermezzo finale in Fedele e altri racconti. L'opera del musicista tedesco ritorna nelle Poesie (Milano, Baldini e Castoldi, I908), IN PARTICOLARE NELLA VERSIONE ITALIANA DEL «PEZZO FANTASTICO», OP. I2, IN DER NACTH (pp. 347-349), e in Leila (1910), in cui il capitolo tredicesimo reca il titolo «Aveu», proprio in riferimento al celebre brano pianistico del compositore ( $« \mathrm{Mi}$ chino, bevo il profumo ch'ella usa e che la carta serba ancora, che mi fa dolere il petto come la divina musica di "Aveu" di Schumann, che, suonata da lei, mi fece dolere in un modo tanto dolce fino alle braccia e ai polsi»).

62. Nella lettera-dedica, Fogazzaro accenna, di passaggio, alla sua trascorsa amicizia con miss Ellen Starbuck, di cui ha perso traccia, e fa appello al «lettore gentile», se può, di dargli sue notizie qualora ne fosse a conoscenza. Lo scrittore aveva conosciuto l'americana Ellen Starbuck durante una vacanza a Lanzo d'Intelvi, nel 1883; la giovane donna divenne ben presto una sua assidua corrispondente e confidente, nonché musa ispiratrice, almeno in parte, per la figura di Violet Yves de Il mistero del poeta (1888). L'episodio narrato nella dedica è parzialmente vero. Nel 1884, durante un viaggio nella Riviera Ligure, lo scrit- 


\section{A la señora Bice Mannellir3}

\section{Señora,}

Puesto que este no es su verdadero nombre y solo usted comprenderá, entre los que aún vivimos, que aqui, dentro de un manto de poesía, oculto el doloroso drama de su pobre y santa hija, me atrevo a decir a quien lee unas palabras de doña Cecilia, por la piedad y la veneración que sentí por ella.

Muchos años pasaron desde el día en que me encontré por vez primera con su juvenil $y$ dulce rostro, vestida de luto, con los grandes ojos sombreados de pio recogimiento, como si caminara entre los pilares de un templo oscuro con lámparas encendidas en el lejano fondo. Hablaba poco, en voz baja y suave, sin abrir nunca el corazón. De vez en cuando se encendía la luz en su mirada y sonaba, en su voz, un tesoro escondido; pero, lo confieso, sus gélidos silencios y las inexplicables indiferencias me convencieron cierta vez de que esa

tore visitò anche la Costa Azzurra, ma non ebbe modo di incontrare miss Starbuck: «La prefazione del Ritratto mascherato potrebbe quindi rientrare nella finzione, rievocando un incontro che non ebbe luogo, o rievocarne uno che, stranamente, non viene mai citato nelle lettere» (Fogazzaro-STARbuck, p. 107, nota 204). Di fatto, gli assidui contatti epistolari tra Fogazzaro e Ellen Starbuck si interromperanno nel 1898 per riprendere solo molto più tardi, nel 1907 e proprio grazie al «messaggio nella bottiglia» lanciato dalla dedica su Scene. Si veda ancora Fogazzaro-Starbuck, n. 85, (Napoli, 29 marzo 1907), pp. 397-399.

63. alla signora Bice Mannelli.

Signora,

Poiché tale non è il Suo vero nome e Lei comprenderà sola, fra i vivi di oggi, che celai qui, dentro un tessuto di poesia, il doloroso dramma della povera figliuola Sua, santa, oso dire a chi legge una parola di donna Cecilia, per la pietà e la venerazione che n'ebbi.

Anni ed anni passarono dal giorno che mincontrai prima nel giovinetto dolce viso di lei vestita a lutto, nei grandi occhi ombrati di raccoglimento pio come sell'andasse fra i pilieri di un tempio scuro a lampade lucenti nel fondo lontano. Parlava rado, piano e soave senz' aprire il cuore mai. Saliva bene di tempo in tempo nel suo sguardo il lume e nella sua voce il suono di un tesoro nascosto; ma, lo confesso, i gelidi silenzii di lei e le indifferenze inesplicabili mi persuasero un tempo che lume e suono fossero parvenze vane, che donna Cecilia fosse una creatura fredda e vacua, come diceva il mondo.

Nell'inverno che la Sua figliuola passò con Lei a Villafranca sul mare, io pure soggiornai una quindicina di giorni a quell Hôtel de l'Univers dove dimoravano altre amiche mie, le signore Starbuck delle quali mi duole infinitamente aver perduta la traccia e che nomino qui per la speranza di averne notizia da qualche lettore gentile. Si fece una gita insieme alla penisola di S. Giovanni. Là, salendo tra gli ulivi verso il semaforo di Capo Ferrat, si parlò di religione $e$ io dissi a miss Starbuck che il perdono divino intero, come quello di Cristo in croce al buon ladrone, non solamente rimette la pena ma del tutto annienta la offesa, rifluisce nel passato reo, lo distrugge anche nella memoria, rimbianca l'anima super nivem. Soggiunsi che perdonare cosi era degno di un amore onnipotente e che l'amore umano, il femminile sopra tutti, era qualche volta portato a trapassar in certo modo il perdono con un errore sublime, a negar la offesa piu flagrante o a giustificarla, a deificare l'amato, a odiare chi gli apponesse una imperfezione, una colpa. Non miss Ellen ma unaltra signora della compagnia osservò che in certi casi potevo aver ragione ma che se si trattasse di tradimento, nessuna donna di cuore e di cervello si comporterebbe tanto stupidamente. Allora donna Cecilia, tutta accesa in volto, tutta vibrante come nessuno l'aveva vista mai, disse con la sua voce pur sempre soave che l'amore non sarebbe amore se non avesse fede e la fede non sarebbe fede se non credesse contro le apparenze. Lo scatto 
luz y ese sonido eran vanas apariencias, y que doña Cecilia era una criatura fría y vacía, como decía todo el mundo.

En el invierno que su hija pasó con usted en Villafranca, a orillas del mar, yo también me alojé durante quince dias en aquel Hôtel de l'Univers en el que se alojaban también otras amigas mías, las señoras Starbuck, de las cuales lamento infinitamente haber perdido el rastro, y que nombro aquí con la esperanza de tener noticias de ellas a través de algún amable lector. Hicimos una excursión juntos al peñón de San Giovanni. Alli, subiendo entre los olivos hacia el semáforo de Capo Ferrat, hablamos de religión, y yo le dije a la señora Starbuck que el perdón divino total, como el que le dio Cristo al buen ladrón en la cruz, no solo redime la pena, sino que anula completamente la ofensa, se remonta hasta el pasado culposo, e incluso lo destruye en la memoria, purificando el alma super nivem. Añadi que perdonar asi era digno de un amor omnipotente y que el amor humano, y en especial el femenino, tendía en ocasiones a ir más allá del perdón con el error sublime de negar la ofensa más flagrante, de justificarla, de deificar al amado, o de odiar a quien le atribuyera alguna imperfección o una culpa. No fue la señora Ellen, sino otra señora de la compañia, quien observó que en ciertos casos yo podía tener razón, pero que si se trataba de engaño, ninguna mujer se comportaría, ni de corazón ni de cabeza, de una forma tan estúpida. Entonces doña Cecilia, con el rostro completamente encendido, y toda ella agitada como jamás nadie la había visto, dijo con su voz, a pesar de todo siempre suave, que el amor no sería amor si no tuviera fe, y que la fe no sería fe si no creyera a pesar de las apariencias. Esta insólita reacción sorprendió tanto que nadie se atrevió a decir: " $Y$ si la traición es evidente?». La conversación decayó y yo regresé a mi impresión inicial de que doña Cecilia era un tarro cerrado de esencias preciosas, que no decía nada por humildad y por pudor del alma.

insolito sorprese tanto che nessuno osò dire: "e se il tradimento si vede?». La conversazione cadde e io ritornai alla mia prima idea che donna Cecilia fosse un vaso chiuso di essenze preziose, che tacesse tanto per umiltà e pudore dell'anima.

Un giorno le parlai di Ruysbroek che stavo leggendo. Sulle prime mi rispose, al solito, con qualche monosillabo distratto. Proseguii tuttavia ed espressi con calore le mie credenze religiose. Compresi tosto che questa era stata per lei una rivelazione gradita e che l'anima sua si apriva. Non conosceva Ruysbroek nè altri grandi mistici di cui le parlai: S. Giovanni della Croce, la Guyon. La sua cultura era scarsa, la sua concezione religiosa poco elevata intellettualmente ma il cuore che mi si rivelò nelle conversazioni a cui donna Cecilia ritornava ogni momento per interrogarmi circa dubbii di coscienza che la turbavano, era veramente come un usciolino socchiuso del paradiso.

Ella tremava, la gentile anima, di dolore, di amore e di timore, sulla soglia dellinfinito. Viveva nell'al di là prima di scomparirvi. L'angoscia sua segreta era il dubbio di spiacere al Signore e rendersi così indegna di pregare per il suo Carlo. M'interrogò una volta sulle confessioni dei morenti. Forse un altro dubbio angoscioso la rodeva, l'irragionevole dubbio che il povero Carlo non fosse stato in grado di fare una confessione esatta; perchè la sua mente era cattiva col suo cuore, non rifiniva di acuir sottili spine da infiggere in esso. Quest' ansia circa la confessione del marito, Le dica, signora, che sia da pensare delle violente proteste di lei contro un divinato sospetto, e dell'aver voluto che quelle carte ardessero, e dell'affannoso grido col quale cadde a Lei nelle braccia. Basta, vi è stato un segreto fra la Sua figliuola e Dio.

Se il mondo che poco la vide male la giudicò, se fu solamente conosciuta e riverita come una creatura del cielo dai poveri e dagli umili, io sarò di costoro, la mia unirò alla lor voce che più dura e più onora. 
Un dia le hablé de Ruysbroek, al que estaba leyendo. Al principio me respondió, como solía hacer, con algún que otro monosilabo suelto. A pesar de ello insistí y expresé con entusiasmo mis creencias religiosas. Comprobé al momento que para ella resultaba una revelación agradable y que su alma se abría. No conocía a Ruysbroek ni a los demás grandes misticos de los que le hablé, san Juan de la Cruz o Jeanne Guyon. Su cultura era escasa, y su concepto de la religión era intelectualmente poco elevado, pero el corazón que se me reveló en las conversaciones a las que doña Cecilia regresaba a cada momento para preguntarme sobre las dudas de conciencia que la inquietaban, era realmente como una puerta entreabierta del paraíso.

Ella temblaba, con su noble alma, de dolor, de amor y de temor, ante el umbral del infinito. Vivía en el más allá antes de adentrarse en él. Su secreta angustia era la duda de disgustar al Señor y de volverse, por tanto, indigna de rogar por su Carlo. Me preguntó cierta vez por las confesiones de los moribundos. Puede que fuera otra duda angustiosa la que la corroía, la duda irracional de que el pobre Carlo no bubiera estado en condiciones de hacer una confesión exacta; estando su mente, como su corazón, enferma, no paraba de afilar agudas espinas para clavárselas en el corazón. Dígale usted, señora, que esta ansia acerca de la confesión del marido hace pensar en una violenta reticencia ante una sospecha intuida, en su empeño por quemar aquellos papeles y en el desesperado grito con el que se arrojó en los brazos de usted. En definitiva, ha habido un secreto entre su hija y Dios.

Si el mundo que la vio poco la juzgó mal, si tan solo fue conocida y venerada como una criatura del cielo por los pobres y por los humildes, yo seré uno de estos, uniré mi voz a la de ellos, que es la que más perdura y más honra. 


\section{IL RITRATTO MASCHERATO}

PERSONAGGI

Cecilia Mannelli, vedova Festi $\left.\begin{array}{l}\text { Professore Mannelli } \\ \text { Signora Mannelli }\end{array}\right\}$ suoi genitori Cavaliere Francesco Festi, suo cognato Dottor Trechi, notaio

Signora Trechi, sua moglie

Giovanni, domestico

La scena è in casa di Cecilia Festi Mannelli. Rappresenta uno studio di scrittore, ammobigliato $^{1}$ con elegante semplicità. Due librerie, un caminetto col fuoco acceso, un ritratto grande di Cecilia, un tavolino per fumare, parecchie sedie e poltrone, un canapè, una scrivania con una lampadina elettrica spenta e un giornale spiegato fra libri e carte in disordine.

Cecilia, sola, siede alla scrivania di contro alla poltrona vuota dove avrebbe a sedere il padrone dello studio. Veste a lutto pesante. Tiene le braccia incrociate sulla scrivania e la fronte reclinata sul dorso della mano destra. Oltre alla porta di mezzo lo studio ha due porte laterali che mettono l'una nelle stanze di Cecilia, l'altra nella biblioteca. ${ }^{2} \mathrm{Si}$ bussa lievemente alla porta di mezzo.

\section{Cecilia}

(trasalendo e levando il capo)

Chi è?

(si alza in piedi e guarda turbata, lagrimosa, verso l'uscio)

Avanti!

(entra Giovanni)

\section{GiovanNi}

Scusi, venivo per il fuoco.

(accomoda con le molle i tizzi del caminetto)

Se viene qualcuno, la signora riceve?

\section{EL RETRATO DE LA MÁSCARA}

\author{
PERSONAJES
}

Cecilia Mannelli, viuda de Festi $\left.\begin{array}{l}\text { Profesor Mannelli } \\ \text { Señora Mannelli }\end{array}\right\}$ sus padres

Caballero Francesco Festi, su cuñado

Doctor TRECHI, notario

Señora Trechi, su esposa

Giovanni, criado

La escena tiene lugar en la casa de Cecilia Festi Mannelli. Representa el estudio de un escritor, amueblado con elegante sencillez. Dos estanterías, una chimenea con el fuego encendido, un retrato grande de Cecilia, una mesita para fumar, bastantes sillas y butacas, un canapé, un escritorio con una lámpara eléctrica apagada y un periódico abierto entre libros y papeles desordenados.

Cecilia, sola, está sentada en el escritorio, frente a la butaca vacía en la que solía sentarse el dueño del estudio. Viste de luto riguroso. Tiene los brazos cruzados sobre el escritorio y la frente apoyada sobre el dorso de la mano derecha. Además de la puerta central, el estudio tiene dos puertas laterales que conducen, una a las estancias de Cecilia, y la otra a la biblioteca. Alguien llama suavemente a la puerta central.

\section{Cecilia}

(sobresaltándose y levantando la cabeza) ¿Quién es?

(se pone de pie y mira asustada, con lágrimas, hacia la puerta)

¡Adelante! (entra Giovanni)

\section{GiovanNi}

Disculpe, venía por el fuego.

(remueve con las tenazas las brasas de la chimenea)

Si viene alguien, ¿la señora recibe?

1. NARDI 1944: «ammobiliato».

2. biblioteca: qui, come in tutte le altre occasioni in cui il termine compare, in NARDI 1944 risulta trascritto con la maiuscola («Biblioteca»). 


\section{Cecilia (dolcemente)}

$\mathrm{Ma}$, mio Dio, Giovanni, non ve l'ho detto che alle due devono venire mio padre, i fratelli e le sorelle del povero padrone? Non vi ho detto che ricevo questi e non altri? Non vi ho dato anche una nota? Vi prego, Giovanni, cercate di aver un po' di memoria in questi momenti.

\section{GiOvANNI}

Perchè, a dire la verità, signora, la nota si è bruciata mezza.

\section{Cecilia \\ Ma chi l'ha bruciata?}

\section{Giovanni}

Io no, signora. La cameriera dice che non l'ha neanche vista e lo stesso dice la cuoca. Ma non importa, adesso ho inteso: ricevere i signori ch'erano qui con lei ieri, durante...

\section{Cecilia}

Ma no, caro Giovanni, tutti no! E poi c'erano le mie cognate, ma i miei cognati lo dovreste ben sapere che non c'erano, che hanno seguito...

(a voce più bassa)

il povero padrone. Loro, dunque, le mie cognate e il signor professore.

\section{Giovanni}

Sissignora, ho inteso.

(Fa per andarsene)

\section{Cecilia}

Aspettate! Anche il notaio. E nessun altro. Avete capito? Nessun altro.

\section{GIOVANNI}

Chi è, signora, il notaio?

\section{Cecilia}

Credo che sia il dottor Trechi. Fatevi dire il nome. Ve lo dirà lui, del resto, ch’è il notaio. Deve esser qui alle due.

\section{Cecilia (delicadamente)}

Pero, Dios mío, Giovanni, ¿no os he dicho ya que a las dos tienen que venir mi padre, y los hermanos y las hermanas del pobre señor? ¿No os he dicho que los recibiré a ellos y a nadie más? ¿No os he dado incluso una nota? Os lo ruego, Giovanni, tratad de tener un poco de memoria en estos momentos.

\section{GiovanNi}

Es que, a decir verdad, señora, la nota se ha quemado un poco.

\section{Cecilia}

Pero ¿quién la ha quemado?

\section{Giovanni}

Yo no, señora. La camarera dice que ni siquiera la ha visto, y lo mismo dice la cocinera. Pero da igual, ya me he enterado: recibirá a los señores que estaban ayer aquí con usted, durante...

\section{Cecilia}

Que no, querido Giovanni, ja todos no! Y además estaban mis cuñadas, pero no mis cuñados, deberías saberlo de sobra, que se fueron a acompañar a...

(en voz más baja)

... el pobre señor. Así que ellos, mis cuñadas y el señor profesor.

\section{Giovanni}

Sí, señora. He entendido.

(Se dispone a marcharse)

\section{Cecilia}

¡Espere! El notario también; y nadie más. ¿Ha entendido? Nadie más.

\section{Giovanni}

¿Quién es el notario, señora?

\section{Cecilia}

Creo que es el señor Trechi. Pregúntele su nombre. Pero de todas formas se lo dirá él, que es el notario. Tiene que estar aquí a las dos. 


\section{GiovanNi}

Ho inteso.

(Esce)

\section{Cecilia}

(Singinocchia di slancio alla scrivania e vi appoggia le mani congiunte)

Dio Dio Dio! Caro caro caro!

(Singhiozza. Si ode un tocco di campanello interno. Cecilia si alza, sta un momento in ascolto, poi si china, bacia lungamente un manoscritto, posa le labbra sulla lampadina elettrica)

Anche te che l'hai servito!

(Entra Giovanni)

Giovanni

Signora, ci sarebbe la signora Trechi.

\section{Cecilia}

Ma Signore, Giovanni, non vi ho detto...?

\section{Giovanni}

Sissignora, ma siccome è la signora del signor notaio, così ho creduto che forse...

\section{Cecilia}

Andate, dite che mi rincresce ma che non vedo nessuno. Se fosse il notaio che mandasse a dire qualche cosa, lo dica a voi.

È qui, signora.

$$
\text { Giovanni (imbarazzato) }
$$

\section{La signora TRECHI}

(entrando precipitosa a mani giunte)

Mi perdoni, mi perdoni, cara signora! Un momento, un momento solo! Sento quanto sono indiscreta, ma si tratta di una parola, di una sola parola! (guarda Giovanni che non si muove)

\section{Cecilia}

(con dolcezza, ma non senza qualche risentimento nella voce)

Mi rincresce, signora... perchè proprio
Entendido

(Sale)

\section{GiovANNI}

\section{Cecilia}

(Se deja caer arrodillándose ante el escritorio y apoya sobre él las manos juntas)

¡Dios, Dios, Dios! ¡Querido, querido, querido!

(Solloza. Se oye una campanilla dentro de la casa. Cecilia se incorpora, se queda un momento a la escucha, vuelve a inclinarse $y$ besa un manuscrito durante un rato; posa sus labios sobre la lámpara eléctrica)

¡A ti también, que le has servido! (Entra Giovanni)

\section{Giovanni}

Señora, la señora Trechi está aquí.

\section{Cecilia}

Pero, por Dios, Giovanni, ¿acaso no le he dicho...?

\section{Giovanni}

Sí, señora, pero como es la esposa del señor notario, entonces me ha parecido que tal vez...

\section{Cecilia}

Vaya y dígale que lo siento, pero que no recibo a nadie. Si fuera porque el notario manda a decir algo, que se lo diga a usted.

\section{Giovanni (en apuros)}

Está aquí, señora.

\section{La señora TRECHI}

(entrando precipitadamente con las manos juntas)

¡Perdóneme, perdóneme, querida señora! ¡Es solo un momento, es solo un momento! Siento ser tan indiscreta, pero se trata solo de una palabra, una sola palabra! (Mira a Giovanni, que no se mueve)

\section{Cecilia}

(Con delicadeza pero no sin un cierto resentimiento en la voz)

Lo siento, señora... porque de verdad que 
non vedo nessuno... Lei capisce... non potrei...

(La signora Trechi guarda Giovanni che alla sua volta interroga con gli occhi la padrona)

Cecilia (in tono rassegnato)

Andate, Giovanni.

(Giovanni esce)

La signora TRECHI (ansante)

Si tratta di una cosa terribile, signora.

Non so... dica presto.

\section{Cecilia (indifferente)}

\section{La signora TRECHI}

Io ho i miei genitori e i miei fratelli in una posizione tristissima. Ne avranno forse anche colpa ma insomma sono genitori e fratelli! Mio marito per un certo tempo li ha aiutati e poi non ha voluto più, mi ha proibito anche a me di far più niente per loro. Gli ho disobbedito una volta e lo ha saputo, c'è stata una scena spaventosa, voleva cacciarmi di casa. Un anno fa questa povera gente aveva bisogno di cinquecento lire per evitare certe cose tristissime, bruttissime. Non le trovavo ${ }^{3}$ da nessuna parte e io non le avevo. Dio mio, come si fa? Siccome vedevo qualche volta Suo marito che veniva dal mio per affari e sapevo ch'era ricco, ch'era tanto generoso, $\mathrm{mi}$ feci coraggio e lo pregai di prestarmi quel denaro che gli avrei poi restituito un po' per volta. Egli fu così buono da prestarmelo e io gli rilasciai un'obbligazione. Mi feci promettere il segreto assoluto, sa, e mi figuro che non avrà parlato neppure con Lei. Cinque giorni sono, lo stesso giorno, credo, che cadde ammalato, ho finito di restituirgli il denaro, sulle scale di casa mia. Egli non aveva con sè, naturalmente, la mia obbligazio- no recibo a nadie... Usted lo entiende... no podría...

(La señora Trechi mira a Giovanni, quien a su vez interroga con la mirada a su ama)

\section{Cecilia (con tono de resignación)} Salga, Giovanni.

(Giovanni sale)

La señora TRECHI (jadeante)

Se trata de una cosa terrible, señora

Cécilia (con
No sé... dígalo ya.

La señora TRECHI

Tengo a mis padres y a mis hermanos en una situación penosa. Es posible que tengan algo de culpa, ipero al fin y al cabo son mis padres y mis hermanos! Mi marido, durante un tiempo les estuvo ayudando, pero después ya no quiso hacerlo más, e incluso me prohibió a mí que hiciera algo por ellos. Una vez le desobedecí y se enteró; me armó una escena espantosa, y quería echarme de casa. Hace un año esta pobre gente necesitaba quinientas liras para evitar ciertos asuntos muy tristes, horribles. Yo no las tenía y no las podía conseguir de ningún sitio ¡Dios mío! ¿qué se podía hacer? Como yo veía de vez en cuando a su marido cuando venía a ver al mío por asuntos de negocios, y como sabía que era rico y muy generoso, me armé de valor y le rogué que me prestara ese dinero, que yo le devolvería poco a poco. Él fue tan bueno que me lo prestó, y yo le extendí un pagaré. Le pedí que me prometiera el más absoluto secreto, ya sabe, así que me imagino que ni siquiera se lo habrá dicho a usted. Hace cinco días, creo que fue el mismo día que se puso enfermo, terminé de devolverle el dinero, en las escaleras

3. Lettura 1902: «Non le trovavano». Nonostante questa lezione ci sembra che concordi meglio con il senso della frase, nel dubbio abbiamo rispettato la forma presente in SCENE 1903.

4. LetTura 1902: "portarmela, che poi fu». 
ne e mi promise di portarmela, che, poi $^{4}$ fu impossibile. Mi disse che la teneva nella scrivania del suo studio. M'indicò anche il posto preciso. So che fra poco deve venir qua mio marito a prendere il testamento, proprio nella scrivania dello studio; me l'ha detto lui mezz'ora fa che ne aveva l'incarico dal cavalier Festi, suo cognato. Capisce, signora, se vede l'obbligazione, col carattere di mio marito, sarà la rovina mia e della mia famiglia. E come è possibile che non la trovi? Farà passare tutte le carte, certo. Lo studio è questo, vero? Allora la scrivania è quella lì. La supplico, la scongiuro, signora, se Lei mi dà la chiave, in due minuti Lei ha la bontà di andar ad avvertire che non entri nessuno, io apro, prendo la carta, chiudo, Le rendo la chiave e fuggo. Se lo incontro gli dico che sono venuta per le condoglianze.

\section{Cecilia}

Ma io non l'ho, la chiave. L'ho data a mio padre l'altra sera e mio padre deve averla consegnata subito a mio cognato.

\section{La signora TRECHI}

Ah mio Dio! A quale Suo cognato? Al cavalier Francesco, m’immagino. Dio, se avessi tempo! Lo conosco tanto il cavalier Francesco. Non potrebbe, signora, far rimandare a domani? Lei è ancora così scossa, poveretta, così sofferente. Ieri il funerale, oggi...

\section{Cecilia (si allontana dalla signora Trechi,} quasi mal sopportando questi compianti)

La prego, La prego, La prego. Scusi, non posso rimandare, non dipende da me, non posso proprio, scusi. Guardi che Suo marito sarà qui subito.

\section{La signora TRECHI}

Provi, signora! Dica una parola! de mi casa. Lógicamente, él no llevaba encima mi pagaré y me prometió que me lo traería, lo cual después fue imposible. Me dijo que lo tenía en el escritorio de su despacho; incluso me indicó el sitio exacto. Sé que dentro de un momento mi marido tiene que venir aquí a recoger el testamento, precisamente en el escritorio del despacho; me lo ha dicho él mismo hace media hora, que se lo había encargado el caballero Festi, su cuñado. Entiéndalo, señora, que si ve el pagaré, con el carácter que tiene mi marido, será la ruina, mía y de mi familia. ¿Y cómo no lo va a encontrar? Revisará todos los papeles, como es normal. El despacho es este, ¿¿verdad?, así que este es el escritorio. Se lo suplico, se lo ruego, señora, si usted me deja la llave, en dos minutos, mientras usted tiene la bondad de ir a advertir que no entre nadie, yo abro, cojo el papel y cierro, le devuelvo la llave y desaparezco. Y si me lo encuentro le digo que he venido a darle el pésame.

\section{Cecilia}

Pero es que yo no tengo la llave; se la di a mi padre la otra noche y mi padre se la debió dar en seguida a mi cuñado.

\section{La señora TRECHI}

¡Ay, Dios mío! ¿A cuál de sus cuñados? Al caballero Francesco, me imagino. ¡Dios, si tuviera tiempo! Conozco bien al caballero Francesco. ¿ No podría, señora, aplazarlo hasta mañana? Usted todavía está $\tan$ afectada, pobre, tan dolida. Ayer fue el funeral, y hoy...

\section{Cecilia (Se aparta de la señora Trechi, soportando apenas estos lamentos)}

Se lo ruego, se lo ruego, se lo ruego, perdóneme, pero no puedo aplazar nada, no depende de mí; de verdad que no puedo, lo siento. Y tenga cuidado, que su marido estará aquí enseguida.

\section{La señora TRECHI}

¡Inténtelo, señora! ¡Diga algo, una palabra! 
Cecilia

Ma no, cosa vuole che dica? Che ragione vuole che trovi?

\section{La signora TRECHI}

Senta, almeno cerchi che mio marito non frughi tanto, non guardi tanto! Ci sarà anche il cavalier Francesco?

\section{Cecilia}

Sì.

\section{La signora TRECHI.}

Ah! E allora, non potrebbe Lei fare in modo che invece di mio marito aprisse lui?

\section{Cecilia}

Questo sì, se sarà possibile.

(Saluta del capo)

La signora TRECHI

Grazie, lo faccia, lo faccia! E mi perdoni! (Esce)

(Cecilia preme, dopo un momento di attesa, il bottone del campanello elettrico. Entra Giovanni).

\section{GiovanNi}

Comandi.

\section{Cecilia}

È uscita?

\section{GiovanNI}

Sissignora.

\section{Cecilia}

Ma perchè l'avete fatta entrare?

\section{Giovanni}

Scusi, signora. Io veramente, siccome Lei ha detto del signor notaio e anche la signora ha detto: «dite' che c'è la moglie del notaio" son ${ }^{6}$ venuto a prendere gli ordini e intanto la signora, invece di

\section{Cecilia}

¡Que no! ¿Qué quiere que diga? ¿Qué excusa quiere que encuentre?

\section{La señora TRECHI}

Mire, ¡trate al menos de que mi marido no hurgue demasiado, que no mire mucho! ¿Va a estar también el caballero Francesco?

Sí.

\section{Cecilia}

\section{La señora TRECHI}

¡Ah! Y entonces ¿no podría usted hacer que en vez de mi marido sea él quien abra los cajones?

\section{Cecilia}

Eso, si es posible, sí.

(La despide con un gesto de la cabeza)

La señora TRECHI

¡Gracias! ¡Hágalo, hágalo! ¡Y perdóneme! (Sale)

(Cecilia espera un momento y pulsa el botón del timbre eléctrico. Entra Giovanni)

\section{GiOvANNI}

Mande

\section{Cecilia}

¿Se ha marchado?

\section{Giovanni}

Sí, señora.

\section{Cecilia}

Pero, ¿por qué la ha dejado entrar?

\section{GIOvANNI}

Lo lamento, señora. Yo, en realidad, como usted me habló del señor notario, y como además la señora dijo: «diga que es la mujer del notario", vine a que usted me ordenara, y mientras tanto la señora,

5. NARDI 1944: «ha detto: "Dite"».

6. NARDI 1944: «notaio», son". 
aspettare, non so che signora sia, mi ha seguito.

\section{Cecilia}

Bene, vi raccomando, quelli che ho detto e nessun altro, nessuno, nessuno! E se insistono, non venite a prender ordini, insistete anche voi che se ne vadano.

Sissignora, ho inteso.

\section{GiovanNi}

(Esce)

\section{Cecilia (sola)}

Dio mio, come può affannarsi tanto, quella donna? Come sarei felice, io, se egli potesse cacciarmi di casa! - Ah Signore, almeno lo trovassero subito questo testamento! Almeno non mettessero tanto le mani $^{7}$ nelle sue carte! Forse non sono stata sincera, io, con quella povera donna.

(Si ode un tocco di campanello). Saranno qui, adesso. - Forse le ho promesso di guardare che il notaio non frughi come se avessi pietà di lei e invece non è vero, il mio orrore è di quelle mani che prenderanno la sua chiave, che apriranno la sua scrivania. Oh Signore, Signore, Signore!

(Entra Giovanni)

\section{GiOvanNi}

Il signor cavalier Francesco.

(Entra Francesco Festi. Cecilia gli va incontro, gli stende le mani in silenzio. Francesco le prende le mani, l'attira a sè, la bacia in fronte. Nessun altro saluto è scambiato).

\section{Francesco (a voce bassa)}

Temevo d'essere in ritardo.

$$
\text { Cecilia (colla stessa voce) }
$$

Io non so mai, adesso, che ore sono. en vez de esperar, que no sé qué clase de señora es, me siguió.

\section{Cecilia}

Bueno, os lo ordeno: solo los que le he dicho y nadie más, ¡nadie nadie! Y si insisten, no venga a pedirme órdenes, insista usted en que se vayan.

\section{GiOvanNI}

Sí, señora, entendido.

(Sale)

\section{Cecilia (sola)}

¡Dios mío! ¿Cómo puede ponerse así de nerviosa esta señora? ¡Qué feliz sería yo, si él pudiera echarme de casa! - ¡Oh, Señor, si por lo menos encontraran rápido ese testamento! ¡Si por lo menos no hurgaran tanto entre sus papeles! Creo que no he sido sincera con esa pobre mujer.

(Se oye un timbre).

Parece que ya están aquí - Quizá le haya prometido preocuparme de que el notario no hurgue entre los papeles, como si sintiera lástima por ella, y sin embargo no es verdad; lo que me horroriza son esas manos que cogerán su llave y que abrirán su escritorio. ¡Oh, señor, señor, señor!

(Entra Giovanni)

\section{El caballero Francesco.}

\section{GiovanNi}

(Entra Francesco Festi. Cecilia le sale al encuentro y le tiende las manos en silencio. Francesco le coge las manos, se la acerca y le besa la frente. No se intercambian más saludos).

\section{Temía llegar tarde. \\ Cecilia (con igual voz) \\ Yo ya no sé ni qué hora es.}

Francesco (en voz baja)

7. È stato corretto un evidente refuso, presente solo in SCENE 1903: «la mani» > «le mani». 
Francesco (guarda l'orologio)

Le due non sono ancora suonate, mancano cinque minuti.

(Pausa. I due siedono, lontani l'uno dall'altro).

\section{Francesco}

Pietro non viene mica, sai. Neppure Valentina. Neppure Maria. Dicono ch'è inutile. Vengo io per tutti. E papà?

\section{Cecilia}

Viene certo, perchè non ha mandato a dir niente.

(Pausa)

Francesco (sempre a voce bassa)

È stata una dimostrazione immensa, ieri. Proprio straordinaria.

(Cecilia si copre il viso con le mani. Nuova pausa)

Hai visto i giornali di stamattina?

(Cecilia scuote il capo silenziosamente)

Son pieni di articoli e tutti belli. Te li ho portati.

(Cava dei giornali. Cecilia non fa segno di ringraziare, nè di prenderli)

Li metto qui, sulla scrivania. Quando crederai leggerai.

(Pausa).

L'ultima volta che l'ho veduto in piedi è stato qui, proprio qui. Mercoledì scorso, alle cinque. Si sentiva già male, qui c'erano quindici Réaumur ${ }^{8}$, io soffocavo e lui aveva freddo, stava al caminetto. Ricordo che l'ho sgridato, anzi, e lui si è seccato, si è inquietato più del ragionevole. Non ne ho fatto caso, sai che il povero Carlo, qualche volta, era piuttosto suscettibile.

\section{Cecilia (vivacemente)}

No, no, non è vero, mai non era suscettibile, avrebbe dovuto perdere la pazienza tutti i giorni con me, non la perdeva mai.
FRANCESCO (mira el reloj)

Todavía no han dado las dos, faltan cinco minutos.

(Pausa. Los dos se sientan, lejos uno del otro).

\section{Francesco}

Pedro no viene, ¿sabes? Y Valentina tampoco, ni Maria. Dicen que no hace falta. Yo vengo por todos. ¿Y papá?

\section{Cecilia}

Seguro que viene, porque no ha mandado a decir nada.

(Pausa)

Francesco (siempre en voz baja)

Fue una demostración inmensa la de ayer. Realmente extraordinaria.

(Cecilia se cubre el rostro con las manos. Nueva pausa)

¿Has visto los periódicos de esta mañana?

(Cecilia sacude la cabeza en silencio)

Están llenos de artículos, a cual más bonito. Te los he traído.

(Saca unos periódicos. Cecilia no hace señales de agradecerlo, ni de cogerlos)

Los dejo aquí, en el escritorio. Cuando te apetezca los lees.

(Pausa).

La última que lo vi de pie fue aquí, justo aquí. Fue el miércoles pasado, a las cinco. Ya se sentía mal; aquí hacía quince grados Réaumur, yo me moría de calor y él tenía frío, pegado a la chimenea. Me acuerdo de que incluso lo reñí, y él se enfadó, y se puso más nervioso de lo normal. No le hice caso, ya sabes que el pobre Carlo, a veces, era bastante susceptible.

\section{Cecilia (enérgica)}

No, no, no es verdad, él no era nunca susceptible; debería haber perdido la paciencia conmigo todos los días, y no

8. Réaumur: unità di misura della temperatura, introdotta nel 1732 da René-Antoine Ferchault de Réaumur (1683-1757). Un grado Celsius $\left({ }^{\circ} \mathrm{C}\right)$ corrisponde a 1,25 gradi Réaumur $\left({ }^{\circ} \mathrm{r}\right)$, pertanto la temperatura registrata nella stanza $\left(15^{\circ} \mathrm{r}\right)$ è di $18,75^{\circ} \mathrm{C}$.

9. Lettura 1902: «suscettibile...». 
Anche qui, quante volte mentre lui lavorava, non son venuta a leggere promettendo di tacere e poi ogni momento era una domanda, anche sciocca tante volte, per farmi spiegare una cosa o l'altra, perchè capisco così poco! $\mathrm{E}$ lui era sempre buono, mi rispondeva sempre. (Pausa)

\section{Francesco}

Sai che non mi posso toglier dal cuore quella romanza che ci hai cantato martedì sera, proprio martedì sera...

\section{Cecilia (esaltandosi)}

Non dirmi, non dirmi questa cosa perchè l'ho sempre anch'io qui alla gola! ${ }^{10} \mathrm{Mi}$ pare di averla chiamata io la morte! E gli piaceva tanto! Dopo che sei andato via tu me l'ha fatta ripetere due volte e poi mercoledì mattina l'ha canterellata non so quante volte lui; diceva di non potersene liberare:

La prima tu mi dài cagion di duol Ma passa il cor. ${ }^{11}$

Ah perchè poi parevano fatte apposta per lui e per me tutte quelle romanze dell' «Amor di donna».

(Convulsa, con gli occhi sbarrati, pieni di affanno).

Francesco, Francesco, ti pare che l'abbia chiamata io, la morte? Lo pensi anche tu?

FRANCESCO

(si alza, va a lei con le mani tese)

Oh, ma Cecilia! Cara Cecilia! Cosa dici? la perdía nunca. Incluso aquí, mientras estaba trabajando, cuántas veces me venía a leer prometiéndole que estaría callada, y después a cada momento le preguntaba algo, incluso alguna tontería, para que me explicara esto o aquello, iporque yo sé tan pocas cosas! Y él siempre era bueno, me respondía siempre.

(Pausa)

\section{FRANCESCO}

¿Sabes que no me puedo quitar de la cabeza esa canción que nos cantaste el martes por la noche, justo el martes por la noche...?

\section{Cecilia (poniéndose nerviosa)} ¡No me lo digas, no me digas eso, porque yo también la tengo en la cabeza! ¡Me parece que fui yo quien llamó a la muerte! ¡Y le encantaba! Después de que tú te marcharas me hizo repetírsela dos veces, y después, el miércoles por la mañana, la canturreó no sé cuántas veces; decía que no podía quitársela de la cabeza:

La prima tu mi dài cagion di duol Ma passa il cor.

¡Ah! Es que parecían hechas a propósito, para él y para mí, todas esas canciones del "Amor de mujer».

(Crispada, con los ojos fuera de las órbitas, llenos de pesadumbre).

Francesco, Francesco, ¿tú crees que fui yo quien invocó a la muerte? ¿Tú también lo crees?

\section{FRANCESCO}

(Se levanta y se le acerca con las manos extendidas)

¡Oh, pero Cecilia! ¡Mi querida Cecilia! ¿Qué estás diciendo?

10. l'ho sempre anch'io qui alla gola!: "Avere in gola. Per i cantanti è il saper cantare ancora un pezzo" (Policarpo Petrocchi, Nòvo dizionàrio universale della lingua italiana, Milano: Treves, 1887-1891, s. v. gola).

11. Si veda n. 21. 
(Entra Giovanni)

Giovanni

Il signor professore.

\section{Cecilia}

Papà!

(Fa un gesto a Francesco come per dirgli di troncare e va incontro al professor Mannelli che entra. Si abbracciano in silenzio, lungamente. Poi Mannelli e Francesco si stringono la mano, pure in silenzio)

\section{Mannelli}

Se vuoi dare un bacio a mammà è nel tuo salotto. Qua non viene. Anzi devi scusarmi se ho perduta un po' la pazienza con Giovanni che faceva difficoltà a lasciarla entrare perchè tu non gliel'avevi detto.

\section{Cecilia}

Vado.

(Esce)

\section{MANNELLI}

(ansioso e commosso, a Francesco)

Cosa Le pare di Cecilia? Come La trova?

FRANCESCO

(con agitazione improvvisa)

Senta, ora ch'è uscita. Io devo assolutamente fare una cosa; devo aprire la scrivania e prender fuori delle carte prima che ritorni lei e prima che venga Trechi.

\section{Mannelli (meravigliato)}

Delle carte? Non so, dico... faccia... forse potrà dirmi... mica per niente... la ragione... cosa... non so, infatti...

\section{Francesco}

Allora, Le dirò. Già non è il momento di far cerimonie, questo. Ho visto qui, cinque minuti fa, la signora Trechi.
(Entra Giovanni)

El señor profesor.

\section{GiOvANNI}

\section{Cecilia}

¡Papá!

(Hace una señal a Francesco como para decirle que dejen ese tema y sale al encuentro del profesor Mannelli, que entra. Se abrazan en silencio, durante un rato. Después Mannelli y Francesco se estrechan la mano, también en silencio)

\section{Mannelli}

Si le quieres dar un beso a tu madre está en el salón; aquí no va a venir. Es más, tienes que perdonarme si he perdido un poco la paciencia con Giovanni, que ha puesto reparos para dejarla pasar porque tú no se lo habías dicho.

Voy.

\section{Cecilia}

(Sale)

\section{MANnelli \\ (ansioso y conmovido, a Francesco)}

¿Cómo ve a Cecilia? ¿Cómo la encuentra?

\section{Francesco}

(con una repentina agitación)

Mire, ahora que ha salido: tengo que hacer una cosa imperiosamente; tengo que abrir el escritorio y sacar unos papeles antes de que ella vuelva y de que venga Trechi.

\section{Mannelli (asombrado)}

¿Unos papeles? Pues, no sé,... hágalo... quizá pueda decirme... no es por nada... el motivo... el qué... no sé, la verdad.

\section{Francesco}

Bueno, le diré. No es el momento para andarse con rodeos. Hace cinco minutos he visto aquí a la señora Trechi. 
Mannelli (trasalendo)

Come? La Trechi ha avuto 1'impudenza ${ }^{12}$ di venir qua? Per che fare?

\section{Francesco}

Ecco, io non so se sia stata proprio qui. Lei non me lo ha detto e io non gliel'ho domandato. L'ho trovata in fondo alle scale che mi aspettava. Appena mi ha visto mi ha afferrato. Così, sa, proprio afferrato.

(Entra Giovanni)

\section{Giovanni}

Il signor dottor Trechi.

TRECHI

(entrando, con voce strascicata)

Con permesso.

\section{Mannelli (a Giovanni)}

Avvertite la signora.

(Trechi, Mannelli e Festi si danno gravemente il buon giorno senza stringersi la mano. Giovanni esce).

\section{TRECHI}

Chiedo scusa di aver tardato. Sono stato a un pelo di non poter venire perchè ho incontrato poco lontano da qui la mia signora che si sentiva male. Ho dovuto accompagnarla fino a casa e se avessi ascoltato lei ci sarei rimasto.

\section{FRANCESCO}

Senta, se crede, possiamo rimandare.

\section{TRECHI}

Oh, si figuri!

Francesco

Ma sì, rimandiamo!

\section{TRECHI}

Ma neppure per idea! Sono tranquillissimo. Se guardassi ai nervi di mia moglie!

\section{Mannelli (sobresaltándose)}

¿Cómo? ¿La señora Trechi ha tenido la desfachatez de venir hasta aquí? ¿Para qué?

\section{Francesco}

Bueno, no sé si ha estado justamente aquí. Ella no me lo dijo y yo no se lo pregunté. La encontré al final de las escaleras, esperándome. En cuanto me vio me sujetó; sí, sí, ya ve, me sujetó de verdad.

(Entra Giovanni)

GiovanNI
El señor doctor Trechi.

TRECHI

(entrando, con la voz parsimoniosa)

Con permiso.

Avise a la señora

(Trechi, Mannelli y Festi se dan los buenos dias, con gesto grave, sin darse la mano. Giovanni sale).

\section{TRECHI}

Les pido disculpas por retrasarme. $\mathrm{He}$ estado a punto de no venir porque me encontré cerca de aquí a mi mujer, que se encontraba mal. He tenido que acompañarla a casa y si le hubiera hecho caso me habría quedado allí.

\section{FRANCESCO}

Mire, si quiere podemos aplazarlo.

\section{TRECHI}

¡Eso faltaría!

\section{FRANCESCO}

¡Que sí! ¡Aplacémoslo!

\section{TRECHI}

¡Ni hablar! Estoy muy tranquilo ¡Si le hiciera caso a los nervios de mi mujer!

12. Lettura 1902: «l'imprudenza». 
Conosco la cura, quattro parole secche; scommetto che a quest'ora sta benissimo.

Francesco (a Mannelli)

Scusi, professore. Come Le dicevo, ci sarebbe da vedere questa cosa in biblioteca. Vuol favorire poichè mia cognata non è ancora qui?

\section{ManNelli}

Come crede.

Scusi, dottore.

$$
\text { Francesco (a Trechi) }
$$

(Esce con Mannelli per la porta della biblioteca. Entra Cecilia).

TRECHI (con un profondo inchino) I miei complimenti.

\section{Cecilia (dolcemente)}

Buon giorno. Scusi: mio padre? Mio cognato?

\section{TRECHI}

Sono usciti adesso, per di là.

Cecilia

(aprendo l'uscio della biblioteca)

Papà! Son qui.

Mannelli (di dentro)

Veniamo subito.

(Pausa).

TRECHI (sommessamente)

Un colpo grande.

(Pausa).

La scrivania è questa, non è vero, signora?

Cecilia (con voce rotta)

Sì, questa.

Mannelli (rientrando)

$\mathrm{Mi}$ rincresce, il cavaliere non trova la chiave. Dubitava di averla dimenticata in biblioteca, ma non c'è.
Conozco la cura: cuatro palabras en seco; seguro que a esta hora estará estupendamente.

\section{Francesco (a Mannelli)}

Disculpe, profesor. Como le decía, habría que ver ese asunto en la biblioteca. ¿Le parece bien que pasemos, ya que mi cuñada no está aquí todavía?

\section{Como usted diga.}

Mannelli

\section{FRANCESCO (a Trechi)}

Disculpe, doctor.

(Sale con Mannelli por la puerta de la biblioteca. Entra Cecilia).

TRECHI (con una profunda reverencia) Mis respetos.

\section{Cecilia (con dulzura)}

Buenos días. Perdone, ¿y mi padre? ¿y mi cuñado?

\section{TRECHI}

Acaban de salir, por ahí.

\section{Cecilia}

(abriendo la puerta de la biblioteca)

¡Papá! Estoy aquí.

Enseguida vamos.

\section{Mannelli (desde dentro)}

(Pausa)

\section{TRECHI (en voz baja)}

Ha sido un golpe muy grande

(Pausa)

El escritorio es este, ¿no es verdad, señora? Sí, es este.

$$
\text { Cecilia (con la voz rota) }
$$

\section{ManNelli (regresando)}

Lo siento, el caballero no encuentra la llave. Pensaba que la había olvidado en la biblioteca, pero no está. 
(Sopraggiunge il cavalier Francesco).

Francesco.

Me ne rincresce tanto, non c'è proprio. Ho paura che bisognerà rimandare per forza.

\section{Cecilia}

Io l'ho data a papà l'altra sera, la chiave.

\section{Mannelli}

E io l'ho data al cavaliere la sera stessa. Si capisce che 1'avrà lasciata a casa.

\section{FRANCESCO}

Dev'essere così. Io sto molto lontano e poi, nell'incertezza... Mi pare che si possa benissimo rimandare a domani alla stess'ora.

\section{TRECHI}

Ecco, veramente ho avvertito il Pretore che si sarebbe andati da lui per la lettura verso le due e mezzo, circa. Sarà lì ad aspettarci. Potrei andar io a dirgli la cosa, ma poi domani sono impedito.

(Francesco e Mannelli si appartano con Cecilia).

Francesco (a voce bassa) Se domani proprio non può, ne chiamiamo un altro, ecco. Cosa ti pare?

(Intanto Trechi si è avvicinato alla scrivania, si leva di tasca delle chiavi e ne fa la prova).

\section{Mannelli}

Per me, benissimo.

\section{Cecilia}

Sì, sì, ne chiamiamo un altro, pare anche a me. Adesso glielo dite.

Senta, dottore.

Francesco (voltandosi)

TRECHI

(fa girar una chiave nella toppa).
(Se añade al grupo el caballero Francesco).

\section{Francesco}

Lo siento muchísimo, pero seguro que no está. Me temo que no habrá más remedio que aplazarlo.

\section{Cecilia}

Yo le di la llave a papá la otra noche.

\section{Mannelli}

Y yo se la di al caballero esa misma noche. Se ve que se la ha dejado en casa.

\section{Francesco}

Seguramente. Yo vivo muy lejos y además, en la duda... Me parece que podríamos aplazarlo a mañana, a la misma hora, sin problema.

\section{TRECHI}

Bueno, en realidad yo había avisado al juez de que iríamos a verlo para la lectura hacia las dos y media, más o menos. Estará allí esperándonos. Yo podría ir a decirle lo que ha pasado, pero es que mañana me resulta imposible.

(Francesco y Mannelli se apartan con Cecilia).

\section{Francesco (en voz baja)}

Si realmente no puede mañana, llamamos a otro notario y ya está. ¿Qué te parece? (Mientras tanto Trechi se ha acercado al escritorio, se saca del bolsillo unas llaves y las prueba).

\section{Mannelli}

Por mí, muy bien.

\section{Cecilia}

Sí, sí, llamamos a otro, a mí también me parece bien. Ahora se lo decís.

Francesco (dándose la vuelta) Mire, doctor...

TRECHI

(logra girar la llave en la cerradura). 
Signori, è aperto. Io ho una chiave miracolosa.

(Mostra la chiave e se la rimette in tasca).

\section{Cecilia}

(accorrendo alla scrivania)

E aperto? Aspetti, La prego. Mi perdonerà, vero, se Le dico che vorrei tanto cercarla io questa carta?

( $A$ suo padre e a suo cognato).

Anche voi mi permettete, vero?

Si figuri!

\section{Trechi}

Mannelli e Francesco (a un tempo) No, no, no, no!

Cecilia

Ma perchè, no? Egli non voleva che nessuno le toccasse le sue carte. ${ }^{13} \mathrm{Mi}$ figuro che se le tocco io gli dispiace meno. Fammi questa grazia, Francesco.

\section{MANNELLI}

Cara, ti commovi troppo!

\section{Cecilia (attonita)}

Mi commovo troppo, papà? Tu mi dici che non mi devo commover troppo? Tu credi che se non tocco quelle carte... questa è amara, sai, papà mio...

\section{FRANCESCO}

No, cara, intendilo, tuo padre dice che sarebbe un soprappiù di angoscia superiore $^{14}$ alle tue forze.

\section{MANNELLI}

Ecco, già!

\section{Francesco}

Guarda, farà tuo padre, ch'è tuo padre, oppure farò io che sono il fratello di Carlo.

(a Trechi)
Señores, ya está abierto. Yo tengo una llave milagrosa.

(Muestra la llave y se la mete en el bolsillo).

\section{Cecilia}

(acercándose apresuradamente al escritorio) ¿Está abierto? Espere, por favor. Usted me perdona si le digo que tengo mucho interés en buscar esa carta ¿verdad?, ( $A$ su padre y a su cuñado).

Vosotros también me permitís, ¿verdad?

TRECHI

¡Faltaría más!

Mannelli y Francesco (a la vez) ¡No, no, no!

\section{Cecilia}

Pero ¿por qué no? Él no quería que nadie le tocara sus papeles. Me imagino que si los toco yo le disgustaría menos. Hazme este favor, Francesco.

\section{MANNELLI}

Querida, ¡estás demasiado consternada!

\section{Cecilia (atónita)}

¿Que estoy demasiado consternada, papá? ¿Tú me dices que no debo estarlo tanto? Tú crees que si no toco estos papeles... esto es muy amargo, sabes, padre mío...

\section{Francesco}

No, querida, entiéndelo, tu padre dice que para ti sería una angustia excesiva, superior a tus fuerzas.

\section{MANNELli}

¡Eso es!

\section{FRANCESCO}

Mira, lo hará tu padre, que para eso es tu padre, o lo hago yo, que soy el hermano de Carlo. (a Trechi)

13. NARDI 1944: «le toccasse, le sue carte».

14. NARDI 1944: «angoscia, superiore». 
Scusi, sa, dottore; Lei capirà.

(Trechi fa un gesto di acquiescenza. Francesco apre prontamente il cassetto, si china a leggere).

Ecco, ecco, guarda che combinazione! (Prende una carta e spinge il cassetto a posto).

Eccolo trovato. Era proprio sopra.

\section{MANNELli (contento)}

Bene. Vedi, cara, che non si è rovistato, che non si è toccato niente?

\section{Trechi}

È chiuso o è aperto il testamento?

\section{Francesco}

Chiuso.

\section{TRECHI}

Allora lo apriremo in Pretura. Se credono ci andiamo ${ }^{15}$ subito, sarà anche tardi. Li prego di venire tutti e due con me per udire la lettura.

\section{Mannelli}

È necessario che venga anch'io? Non credo.

\section{TRECHI}

Se venisse farebbe un piacere ${ }^{16}$ al cavalier Festi, non è vero?

\section{Francesco}

Certo.

(a Trechi)

Vuol chiudere il cassetto?

TRECHI

Volentieri ${ }^{17}$.

(chiude)

\section{Cecilia}

Adesso mi pare che possiate lasciare aperto.

15. NARDI 1944: «Se credono, ci andiamo».

16. LetTURA 1902: «farebbe piacere».

17. LetTura 1902: «Volontieri».
Perdone, ya sabe, doctor, usted lo entenderá.

(Trechi hace un gesto de aprobación. Francesco se apresura a abrir el cajón y se agacha para leer dentro).

Aquí está, aquí está, ¡mira qué suerte!

(Coge un papel y cierra el cajón).

Ya lo he encontrado. Estaba justo el primero.

\section{ManNelli (contento)}

Bien. ¿Lo ves, querida, que no hemos removido ni hemos tocado nada?

\section{Trechi}

¿El testamento está abierto o está cerrado?

$$
\text { FRANCESCO }
$$

Está cerrado.

\section{TRECHI}

Entonces lo abriremos en el juzgado. Si les parece nos vamos ahora mismo, que ya debe ser tarde. Les ruego que vengan los dos conmigo, para oír la lectura.

MANNELLI

¿Hace falta que vaya yo? No lo creo

\section{TRECHI}

Si viniera le haría un favor al caballero Festi, ¿no es verdad?

\section{Francesco}

Desde luego.

(a Trechi)

¿Quiere cerrar el cajón?

TRECHI

Con mucho gusto.

(cierra)

\section{Cecilia}

Ahora me parece que ya lo pueden dejar abierto 


\section{TRECHI}

Senta, signora.

(presenta la chiave a Cecilia)

Questa chiave non mi occorre. Io la lascio a lei fino a che avranno trovata l'altra.

Non s'incomodi!

\section{Mannelli}

Cecilia (che ha presa ${ }^{18}$ la chiave) Poichè è tanto gentile, accetto. Ho piacere di averla.

(Trechi e il cavalier Francesco salutano Cecilia ed escono. Mannelli rimane indietro).

\section{MANNELli}

Cecilia, adesso vai da mammà, vero? Stai nel tuo salotto, con lei? Io ritorno appena finito. Anzi, se permetti, lascio qui un libro che m'incomoda un poco. Andiamo, cara. Ti voglio condurre io, da mammà.

\section{Cecilia}

No, papà, ti prego. Invece mandamela qua lei.

ManNelli

Ma perchè? Vieni!

Cecilia

No, no, ti scongiuro; per mammà è lo stesso, io starei sempre qui, giorno e notte.

(Entra la signora Mannelli)

La signora MANNELLI

Sono partiti? Ah, papà è ancora qui.

\section{Mannelli}

Vado. Persuadi tu Cecilia di non restare qui adesso. Ci è stata tutto il giorno, è troppo!

(Esce)

\section{TRECHI}

Mire, señora...

(Le enseña la llave a Cecilia)

Esta llave no me hace falta. Se la dejo a usted hasta que encuentren la otra.

\section{MANNELLI}

¡No tiene por qué molestarse!

Cecilia (que ha cogido la llave)

Ya que es tan amable, la acepto. Me agrada tenerla.

(Trechi y el caballero Francesco saludan a Cecilia y salen. Mannelli se queda atrás).

\section{Mannelli}

Cecilia, ahora vas con mamá, ¿no? ¿Te quedas en el salón con ella? Yo vuelvo en cuanto termine. Es más, si me permites, dejo aquí un libro que me estorba un poco. Vamos, querida, que te quiero acompañar adonde está mamá.

\section{Cecilia}

No, papá, te lo ruego. Mejor tráemela aquí a ella.

\section{MANNELLI}

Pero ¿por qué? ¡Vente!

\section{Cecilia}

No, no, te lo suplico; a mamá le da igual, y yo me quedaría todo el tiempo aquí, de día y de noche.

(Entra la señora Mannelli)

\section{La señora MANNELli}

¿Ya se han marchado? Ah, papá está aquí todavía.

\section{ManNelli}

Me voy. Convence tú a Cecilia para que no se quede aquí ahora. Lleva aquí todo el día, ¡es demasiado!

(Sale) 
La signora MANNELLI

Non vuoi proprio venir via?

\section{Cecilia}

No, mamma, no.

(Come risovvenendosi di qualche cosa)

Ah c'è anche questo; una povera creatura da tranquillare.

Chi? Che creatura?

\section{La signora MANNELLI}

Cecilia

Niente, devo cercar delle carte nella scrivania.

La signora Mannelli

Posso aiutarti?

\section{Cecilia}

Come vuoi. Però è meglio che faccia io.

La signora Mannelli

Senti, cara. E non prenderesti qualche cosa, prima? La tua gente mi ha detto che non hai ancora preso nulla, oggi. Non puoi andar avanti così, Cecilia. Pensa che potresti anche trovarti, chi sa, in uno stato... ${ }^{19}$

\section{Cecilia (interrompendo)}

No, mamma mia, non parlarmene. Dirai che dovrebb'essere un conforto, ma io non lo voglio appunto per questo. I conforti della mia fede, quelli sì; altri no, no, no. E se tu sentissi come sto bene! Non sono mai stata così bene. Cerchiamo questa carta, ora.

(Siede alla scrivania, vi punta $i$ gomiti e stringendosi il viso tra le mani si affisa, come trasognata, nel vuoto, recita con voce fioca):

La prima tu mi dài cagion di duol Ma passa il cor.
La señora MANNELLI ¿Seguro que no quieres venir fuera?

\section{Cecilia}

No, mamá, no.

(Como acordándose de algo)

¡Ah!, y hay otra cosa. Tengo que tranquilizar a una pobre criatura.

La signora Mannelli

¿Quién? ¿Qué criatura?

\section{Cecilia}

No, nada; tengo que buscar unos papeles en el escritorio.

\section{La señora MANNELLI}

¿Puedo ayudarte?

\section{Cecilia}

Como quieras. Pero es mejor que lo haga yo.

\section{La señora MANNELLI}

Mira, hija ¿no quieres tomarte algo antes? Tus sirvientes me han dicho que hoy todavía no has tomado nada. No puedes seguir así, Cecilia. Piensa que podrías encontrarte, quién sabe, en un estado...

\section{Cecilia (interrumpiendo)}

No, mamá, no me hables de ello. Me dirás que debería ser un consuelo, pero yo no lo quiero precisamente por eso. Los consuelos de mi fe, esos sí; otros no, no, ni hablar. ¡Y si tú sintieras lo bien que estoy! ¡Nunca he estado tan bien! Vamos a buscar ese papel, ahora.

(Se sienta en el escritorio, apoya los codos y cubriéndose el rostro con las manos se queda con la mirada perdida en el vació, como traspuesta, y recita con un bilo de voz):

La prima tu mi dai cagion di duol Ma passa il cor. 
Spietato, immerso nel profondo sei

Mortal sopor.

La derelitta guarda intorno a sè

Tutto è squallor

Non ho più amor — vissuto hai tu

Io non son viva più. ${ }^{20}$

Sai, mamma, che l'ho chiamata io la morte?

La signora MANNELLI

Cecilia! Come puoi dire queste cose?

Cecilia

Lo dico e lo penso, mamma. Perchè vedi, lo sai bene, io le cantavo tanto quelle arie dell' Amor di donna di Schumann. Tutte, ma sopra tutte "La mente mia si smarrisce» e questa. L'adoravo, quella poesia, quella musica. Dici di no, mamma mia, che non l'abbia chiamata io, la morte?

La signora MANNELLI

Cecilia! Tu mi hai rimproverate delle superstizioni, qualche volta, colla tua dolcezza. Ma questa cos'è? Dimmi!

\section{Cecilia}

Ti pare una superstizione, mamma? $\mathrm{Ti}$ pare che sia peccato di pensare così ${ }^{21}$ Allora non penserò più così, non penserò più così. Il Signore mi perdonerà perchè non mi è venuto in mente che fosse peccato. E non ne sono mica proprio sicura, sai, ancora. Però, nel dubbio, non bisogna, vero? Non ho mai avuto tanto orrore di far peccati, mamma, come adesso che devo pregar per Lui e prepararmi ad andar con Lui!
Spietato, immerso nel profondo sei

Mortal sopor.

La derelitta guarda intorno a sé

Tutto è squallor

Non ho più amor — vissuto hai tu

Io non son viva più.

¿Sabes, mamá, que fui yo quien llamó a la muerte?

La señora MANNELLI

¡Cecilia! ¿Cómo puedes decir esas cosas?

\section{Cecilia}

Lo digo y lo pienso, mamá. Porque mira, tú lo sabes bien, yo le cantaba muchas veces las arias del Amor de mujer de Schumann; todas, pero sobre todo «Mi mente se pierde» y esta. Yo adoraba esa poesía, esa música. ¿Tú dices que no, madre mía, que yo no he llamado a la muerte?

La señora MANNELLI

¡Cecilia! ¡Tú me has reprochado a veces, con tu dulzura, que tengo supersticiones! ¿Y esto qué es? Dímelo.

\section{Cecilia}

¿Te parece una superstición, mamá? ¿Te parece que es un pecado pensar así? Entonces dejaré de pensar así, dejaré de hacerlo. El Señor me perdonará, porque no se me ha ocurrido que fuera un pecado. Ni siquiera estoy segura todavía de que lo sea. Pero por si acaso no conviene ¿verdad? Nunca he tenido tanto miedo a cometer pecados, mamá, como ahora que tengo que rezar por él y prepararme para irme con él.

20. «Me causas el primer dolor, / Pero me llega al corazón. / Despiadado, inmerso estás en el profundo / y mortal sopor. // La desdichada mira a su alrededor, / Todo es desolación. / Ya no tengo amor - tú has vivido, / Yo ya no estoy viva». Il testo originale è: «Nun hast du mir den ersten Schmerz getan, / Der aber traf. / Du schläfst, du harter, unbarmherz'ger Mann, / Der Todesschlaf. // Es blicket die Verlaßne vor sich hin, / Die Welt ist leer. / Geliebet hab ich und gelebt, ich bin / Nicht lebend mehr» (in Adalbert von CHamisso, Sämtliche Werke, Monaco: Winkler, 1975, vol. I, p. 154).

21. È stato emendato il punto esclamativo presente in SCENE 1903 e NARDi 1944 («cosi!»), perché non giustificato dal senso della frase, ed è stato sostituito con il punto interrogativo («così?»), già presente in LETTURA 1902. 
La signora MANNELLI

Bambina mia, non ne hai mai fatto, tu, dei peccati.

\section{Cecilia}

(coprendosi gli occhi con le mani)

Oh mamma, mamma!

(Pausa)

E intanto mi dimentico ancora della carta. (Apre il cassetto)

La signora MANNELLI

Lascia che ti aiuti.

\section{Cecilia}

Sì, sì, aiutami. Tanto tu non parlerai. Perchè si tratta di un segreto. Bisogna trovare una lettera della signora Trechi.

\section{La signora MANnelLI (scattando)}

Della signora Trechi?

\section{Cecilia}

Sì, della signora Trechi. Una lettera in cui si riconosce debitrice di cinquecento lire che Carlo le aveva prestate.

\section{La signora MANNELLI}

Ma perchè la cerchi adesso?

\section{Cecilia}

Perchè è stata qui lei, poco fa, tutta affannata per la paura che suo marito, facendo passar le carte, qui dentro, la scoprisse. Suo marito non sa niente e guai se sapesse. Voleva levarla lei, ma io non avevo la chiave. Adesso penso di cercarla e di mandargliela perchè si dia pace, povera donna.

\section{La signora MANNELLI}

Cara te, lascia un po' stare. La cercherà papà, quando ritorna. Credo che sarà qui subito.

\section{Cecilia}

No, a papà non lo voglio far sapere. Mi

\section{La señora Mannelli}

Mi niña, pero tú no has cometido nunca ningún pecado.

\section{Cecilia}

(cubriéndose los ojos con las manos)

¡Oh, mamá, mamá!

(Pausa)

$\mathrm{Y}$ a todo esto se me vuelve a olvidar lo del papel.

(Abre el cajón)

\section{La signora MANNELLI}

Deja que te ayude.

\section{Cecilia}

Sí, sí, ayúdame. De todas formas no vas a decir nada, porque se trata de un secreto. Hay que encontrar una carta de la señora Trechi.

\section{La señora MANnelli (sobresaltándose)} ¿De la señora Trechi?

\section{Cecilia}

Sí, de la señora Trechi. Una carta en la que se reconoce deudora de quinientas liras que Carlo le había prestado.

\section{La señora MANNELLI}

Pero ¿por qué la buscas ahora?

\section{Cecilia}

Porque ha estado aquí, hace un momento, muy preocupada por miedo a que su marido, revisando los papeles que hay aquí, la descubriera. Su marido no sabe nada, porque si lo supiera... Quería llevársela ella, pero yo no tenía la llave. Ahora quiero buscarla y mandársela para que se tranquilice, la pobre mujer.

\section{La señora MANnelli}

Querida, deja eso ahora. Ya la buscará papá, cuando vuelva. Creo que volverá ya mismo.

\section{Cecilia}

No, no quiero que papá se entere. Me ha 
pare di aver capito che la signora Trechi gli sia antipatica. Non vorrei che poi facesse delle supposizioni poco caritatevoli sull'uso di quel denaro.

(Leva dal cassetto e porge a sua madre un fascio di carte).

Fa passar queste, tu, intanto. Sarà bene di levar fuori tutto. Ah, Dio mio!

(Fruga nel cassetto per raccogliervi sulla bocca tutte le carte).

Qui in fondo c'è anche...

(Leva una fotografia, sinterrompe, la guar$d a)$.

\section{Cos'è?}

La signora MANNELLI

Cecilia

(a voce bassa, ma non turbata, guardando sempre la fotografia).

Una fotografia. Una signora in toilette ${ }^{22}$ da ballo, con la maschera.

(Pausa)

La signora MANNELLI

(con voce un po' tremante)

Con la maschera? Lascia vedere.

Cecilia (le porge la fotografia)

$\mathrm{E}$ anche delle lettere ci sono. Sarà forse qui la lettera della signora Trechi.

La signora MANNELLI (vibrata) Dammele. Le passerò io. La fotografia non è della Trechi.

\section{Cecilia (tranquilla)}

Non ho mica detto che fosse della Trechi, $^{23}$ io.

La signora MANNELLI

Pareva che lo supponessi.

\section{Cecilia (nervosa)}

Io? Non supponevo niente. Del resto mi pare che sia della Trechi. Non l'ho mai parecido entender que la señora Trechi le resulta antipática. No querría que después hiciera suposiciones poco caritativas sobre el uso de ese dinero.

(Saca del cajón un fajo de papeles y se lo da a su madre).

Ve revisando tú estas, para empezar. Convendrá sacarlo todo ¡Oh, Dios mío!

(Hurga en el cajón para reunir en su abertura todos los papeles).

Aquí en el fondo también hay...

(Saca una fotografía, se queda quieta, la mira).

¿Qué es?

$$
\text { La señora MANNELLI }
$$

\section{Cecilia}

(en voz baja, pero sin alterarse, sin dejar de mirar la fotografia).

Una fotografía. Una señora con vestido de baile, con una máscara.

(Pausa)

\section{La señora MANNELLI}

(con la voz algo temblorosa)

¿Con una máscara? Déjame ver.

\section{Cecilia (le da la fotografia)}

Y también hay unas cartas. Aquí puede que esté la carta de la señora Trechi.

La señora MANNELli (alterada)

Dámelas. Yo las revisaré. La fotografía no es de la señora Trechi.

\section{Cecilia (tranquila)}

Pero si yo no he dicho que sea de la señora Trechi.

\section{La señora MANNELLI}

Parecía que lo supusieras.

\section{Cecilia (nerviosa)}

¿Yo? No suponía nada. Pero la verdad es que me parece que es la señora Trechi.

22. NARDI 1944: «Una fotografia... Una signora in toilette».

23. Lettura 1902: «Non ho mica detto che sia della Trechi». 
vista sorridere, ma deve avere un sorriso così. Sia della Trechi o non sia della Trechi, mamma, per me fa lo stesso.

La signora MANNELlI

S'intende bene. Ti dirò io che fotografia è. È vero che somiglia alla Trechi. Mi ricordo che Carlo l'ha veduta a Milano, in Galleria, un giorno che si passeggiava insieme noi due, mentre tu scrivevi lettere all'albergo. Gli è piaciuta e l'ha comperata. E adesso dammi le lettere, presto.

\section{Cecilia (vibrata)}

Te le darò ma non c'è nessuna premura!

La signora Mannelli

Oh lo so! Dicevo perchè ci sbrigassimo. Me le dai?

(Butta il ritratto sulla scrivania).

\section{Cecilia}

Perchè lo butti via così, quel povero ritratto?

La signora MANNelli

Dammi le lettere, andiamo, facciamo presto, se papà non ha da saper niente. $\mathrm{E}$ tu prendi fuori il resto, intanto.

\section{Cecilia (balzando in piedi)}

Mamma! Tu mi fai male, sai. Perchè io ti capisco, tu hai ${ }^{24}$ dei sospetti, di' la verità!

\section{La signora MANNELLI (atterrita)}

Ma no, non ho sospetti, non ho sospetti!

\section{Cecilia}

(con agitazione crescente e con lagrime)

Sì, sì, tu hai sospetti. Mi fai male, mi fai male, mi fai male! E non è la prima volta che mi fai male. Tu e anche il papà. Sì, anche il papà!

24. LetTura 1902: «Tu ha dei sospetti».
Nunca la he visto sonreír, pero debe tener una sonrisa así. Que sea la Trechi o no, mamá, a mí me da igual.

\section{La señora Mannelli}

Eso ya se sabe. Yo te diré qué fotografía es. Es verdad que se parece a la señora Trechi. Me acuerdo que Carlo la vio en Milán, en la Galería, un día que nosotros dos dábamos un paseo, mientras tú te quedaste en el hotel escribiendo cartas. Le gustó y la compró. Y ahora dame esas cartas, venga.

\section{Cecilia (alterada)}

¡Ya te las doy, pero no hay que tener prisa!

\section{La señora Mannelli}

¡Ya lo sé! Lo decía para que nos diéramos prisa ¿Me las das?

(Arroja el retrato sobre el escritorio).

\section{Cecilia}

¿Por qué tiras de esa forma el pobre retrato?

\section{La señora MANNelLI}

Dame las cartas, vamos, démonos prisa, si no quieres que papá se entere. Y coge todo lo demás, de paso.

Cecilia (poniéndose de pie de un salto) ¡Mamá! Me estás haciendo daño, ¿`sabes? Porque yo me doy cuenta, ¡tú estás sospechando algo!, ¡di la verdad!

La señora MANnelLi (aterrorizada) ¡Que no! ¡no sospecho nada! ¡no sospecho nada!

\section{Cecilia}

(cada vez más nerviosa y con lágrimas) Sí, sí, tú sospechas algo. Me estás haciendo daño, me estás haciendo daño, y no es la primera vez que lo haces. Tú y papá también, sí, papá también. 
La signora MANNELLI Ma no, cara! Ma quietati!

\section{Cecilia (agitatissima)}

Sì, sì, sì! Quante volte l'ho capito che avevate dei sospetti! Non avete mai detto nomi, ma discorsi vaghi me ne avete fatti tanti! "Meglio che Carlo non vada qui, meglio che Carlo non vada là», oppure: "vacci ${ }^{25}$ anche tu, non lasciarlo andar solo" e tante parole così. Credete che non abbia capito? Credete che non $\mathrm{mi}$ abbia fatto male? Non mi conoscete, nè tu nè il papà. Scusa, mamma, non capite, proprio non capite che ferir lui, per me, è cento, è mille volte peggio che ferir me. In passato tacevo. Soffrivo dentro di me, ma tacevo. Adesso ch'è morto, no, non taccio. E come è morto! Con quella dolcezza di parole che mi ha detto, con quella consolazione delle parole che gli ho detto io, col suo Signore nel petto, con il Crocifisso in mano. E tu, mamma, mi vieni fuori, adesso, con questi miserabili sospetti, con queste offese! Sí, sí, tu hai paura che io scopra chi sa cosa! Mamma, mamma, tu non sai com'è il bene che gli voglio io! Tu non puoi capire che se non fosse stato per la religione, per la legge del Signore, io avrei calcolato niente il mio soffrire se un'altra donna meno stupida di me, meno ignorante di me lo avesse potuto rendere felice! ${ }^{26} \mathrm{Se}$ avessi capito che ci fosse qualche cosa, sai quel che avrei fatto? Avrei pregato il Signore di farmi morire e se il Signore mi avesse fatto la grazia sarei morta in pace, tanto in pace, tanto contenta. Non sai che non ho mai potuto intendere come si sia innamorato di me, Carlo? Non ti permetto di offenderlo. Dio mio, mamma, ho paura di dimenticarmi che sei la mia mamma. Scusa, scusa, scusa. Ma tu non le guarderai, quelle lettere; neppure una ne guarderai; e neppure io le guarderò; adesso; mi parrebbe d'insultare il mio
La señora MANNELLI

¡Que no, hija mía! ¡Tranquilízate!

\section{Cecilia (muy nerviosa)}

¡Que sí! ¡que sí! ¡Cuántas veces me he dado cuenta de que sospechábais algo! ¡Nunca habéis pronunciado nombres, pero habéis insinuado el tema de forma ambigua muchas veces! «Mejor que Carlo no vaya por aquí, mejor que Carlo no vaya por allá», o bien, "ve tú también, no lo dejes que vaya solo» y muchas cosas por el estilo. ¿Os creéis que yo no me daba cuenta? ¿Creéis que no me hacía daño? No me conocéis, ni tú ni papá. Perdona mamá, pero no os dais cuenta, para nada, de que hacerle daño a él, para mí, es cien, mil veces peor que hacerme daño a mí. Antes me quedaba callada; sufría dentro de mí, pero me callaba. Ahora que está muerto, ya no me callo. ¡Y cómo se ha muerto! Con esa dulzura de palabras que me dijo, con esa consolación de las palabras que yo le dije, con su Señor en el pecho, con el crucifijo en la mano ¡Y tú, mamá, me vienes ahora con estas sospechas miserables, con estas ofensas! Sí, sì, tú tienes miedo de que yo descubra quién sabe qué cosas. ¡Madre, madre, tú no sabes cómo lo quiero! Tú no puedes entender que si no hubiera sido por la religión, por la ley del Señor, yo no habría dado ninguna importancia a mi sufrimiento si otra mujer menos estúpida que yo y menos ignorante que yo lo hubiera podido hacer feliz! Si hubiera sabido que había algo, ¿sabes qué habría hecho? Le habría rogado al Señor que me dejara morir, y si el Señor me hubiera concedido la gracia habría muerto en paz, muy en paz, muy contenta. ¿Sabes que nunca he podido entender cómo Carlos se pudo enamorar de mí? No te permito que lo ofendas. Dios mío, mamá, tengo miedo de olvidarme de que eres mi madre. Perdona, perdona, pero tú estas

25. NARDi 1944: "oppure: "Vacci"».

26. LetTura 1902: «rendere più felice!». 
caro, il mio amore, il mio tutto dopo Dio. Prendi, mamma mia.

\section{(Le consegna le lettere)}

Va, bruciale, bruciale subito! Se c'è dentro l'obbligazione brucierà ${ }^{27} \mathrm{e}$ in ogni modo io scriverò a quella signora che nessuno l'ha vista e che l'ho distrutta!

La signora MANNELLI (quasi supplichevole, tenerissima) Sì, ma credi, cara...

\section{Cecilia}

Va, va, va, brucia, brucia, brucia! E poichè sai che il ritratto è un ritratto comperato, brucialo pure anche quello, brucia, brucia!

\section{La signora MANNELLI}

Sì, sì, cara.

(Butta le lettere e il ritratto sul fuoco. Cecilia l'ha seguita sin quasi al caminetto)

\section{Cecilia}

Hai bruciato? Hai bruciato tutto?

La signora MANNELLI

(volgendosi a lei)

Sì, sì, tutto.

\section{Cecilia}

Oh, mamma mia!

(Le si getta singhiozzando fra le braccia)

cala la tela cartas no vas a verlas, ni siquiera una, ni yo tampoco. Ahora me parecería insultar a mi amor, a mi querido, a mi todo después de Dios. Cógelas, madre mía (le entrega las cartas)

¡Venga, quémalas, quémalas ahora mismo! ¡Si el pagaré está dentro se quemará y en cualquier caso yo le escribiré a esa señora que nadie lo ha visto y que lo he destruido!

\section{La señora Mannelli}

(casi suplicando, con mucha ternura)

Sí, pero créeme, querida...

\section{Cecilia}

¡Venga, venga, quémalas, quémalas! Y como además sabes que el retrato es un retrato comprado, quémalo también, quémalo, quémalo!

$$
\text { La señora MANNELLI }
$$

Sí, sí, hija mía.

(Tira las cartas y el retrato al fuego. Cecilia la ha seguido hasta prácticamente llegar a la chimenea)

\section{Cecilia}

¿Lo has quemado todo? ¿Todo?

La señora MANNELli

(volviéndose hacia ella)

Sí, sí, todo.

\section{Cecilia}

¡Oh, madre mía!

(Se le echa a los brazos, sollozando)

cae el telón 